\title{
Studying star forming dwarf galaxies in Abell 779, Abell 1367, Coma, and Hercules clusters $\star, \star \star$
}

\author{
O. Vaduvescu ${ }^{1,2,3}$, V. Petropoulou ${ }^{4}$, D. Reverte ${ }^{5,2}$, and V. Pinter ${ }^{3}$ \\ ${ }^{1}$ Isaac Newton Group of Telescopes (ING), Apto. 321, 38700 Santa Cruz de la Palma, Canary Islands, Spain \\ e-mail: ovidiuv@ing.iac.es \\ 2 Instituto de Astrofísica de Canarias (IAC), C/ Vía Láctea s/n, 38205 La Laguna, Tenerife, Spain \\ 3 The University of Craiova, School of Doctoral Sciences, Str. A. I. Cuza nr. 13, 200585 Craiova, Romania \\ 4 Centro di Ateneo di Studi ed Attività Spaziali “Giuseppe Colombo" (CISAS), University of Padova, Via Venezia 15, \\ 35131 Padova, Italy \\ 5 GRANTECAN S.A., Centro de Astrofísica de La Palma, C/ Cuesta de San José s/n, 38712 Breña Baja, La Palma, Spain
}

Received 27 March 2018 / Accepted 1 June 2018

\begin{abstract}
Context. We continue to study star formation in dwarf galaxies located in nearby clusters.

Aims. Known physical and chemical relations outlining the formation and evolution of dwarfs is compared in different environments, including the Local Volume (LV) and some nearby clusters studied previously.

Methods. We used the TNG telescope for four nights in 2010 to acquire deep near-infrared imaging in $K^{\prime}$ of 45 star forming dwarf galaxies located in the Abell 779, Abell 1367, Abell 1656 (Coma), and Abell 2151 (Hercules) clusters.

Results. Surface photometry was approached based on past experience by using the sech law to account for the outer old stellar contribution plus a Gaussian component to model the inner starburst, proving the blue compact dwarf (BCD) classification of most targets. Sech central surface brightness, semimajor axis, sech, and total apparent magnitude were measured, allowing to estimate size, absolute luminosity and mass for all targets.

Conclusions. The physical correlations between size, central brightness, and NIR luminosity appear to hold, but previously known linear fits break above $M_{\mathrm{SK}}=-19$ for Abell 779, Abell 1367 and especially for Hercules, while the dwarf fundamental plane (FP) is probed by only half cluster members, suggesting harassment by the denser cluster environments. Nevertheless, the chemical relations between the oxygen abundance, luminosity, gas mass, baryonic mass, and gas fraction in a closed box model are probed by most members of the four studied clusters, and the starburst grows linearly with the $K^{\prime}$ luminosity.
\end{abstract}

Key words. galaxies: dwarf - galaxies: star formation - galaxies: photometry - galaxies: clusters: general galaxies: fundamental parameters - galaxies: abundances

\section{Introduction}

Star forming (SF) dwarf galaxies comprise dwarf irregulars (dIs) and blue compact dwarfs (BCDs), which probably represent the first galaxy entities to be formed at different times in the Universe. While dIs are mostly observed as more quiescent entities in isolation or loose groups including the Local Group (e.g. Mateo 1998; Karachentsev et al. 2013), BCDs are encountered mostly in denser environment and galaxy clusters and harbour starburst possibly triggered by strong environmental influences (e.g. Vilchez 1995; Meyer et al. 2014). The physical and chemical properties of dIs and BCDs are linked, suggesting that BCDs represent the outcome of dIs or just some temporary stages of dIs encountered in outburst (e.g. Thuan et al. 1985).

According to hierarchical clustering models, SF dwarf galaxies are believed to be the building blocks of larger galactic structures, being important probes for studying the evolution of matter in near-primordial state (White et al. 1991; Kauffmann et al. 1993). By comparing BCDs in isolation with

\footnotetext{
* Based on observations made with the Telescopio Nationale Galileo (TNG) in La Palma.

$\star \star$ Reduced images as FITS files are only available at the CDS via anonymous ftp to cdsarc.u-strasbg.fr $(130.79 .128 .5)$ or via http://cdsarc.u-strasbg.fr/viz-bin/qcat?]/A+A/616/A165
}

those located in looser and denser clusters, one could study the environmental influences upon star formation in the universe.

The best method to trace star formation is via deep $\mathrm{H} \alpha$ surveys, and Iglesias-Parámo pioneered such work in galaxy clusters (Iglesias-Paramo \& Vilchez 1997, 1999; Iglesias-Paramo 1998). In particular, this author presented a deep $\mathrm{H} \alpha$ survey of the central regions of the nearby clusters Coma (Abell 1656 denoted A1656) and A1367 (Iglesias-Paramo et al. 2002, 2003). Continuing this work, Reverte et al. (2007), Reverte (2008) studied five more Abell clusters: A779, A634, A400, A539 and A2666, all of them being visible from the north and located at the same distance (about $100 \mathrm{Mpc}$ ), thus belonging to a semi-spherical shell of the local Universe. Petropoulou et al. (2011) performed spectroscopic observations and collected existing SDSS DR8 data for a large sample (781 objects) of lowmass SF galaxies in seven nearby clusters in order to investigate the influence of the environment on the formation and chemical evolution of SF galaxies (Petropoulou 2012; Petropoulou et al. 2012). This sample of local Universe clusters span a variety of physical properties (such as mass, X-ray luminosity and evolutionary state) and these authors found that the intracluster medium plays a relevant role on the chemical evolution of lowmass SF galaxies located in massive clusters. Part of a related project, Cedres et al. (2009) presented a deep $\mathrm{H} \alpha$ survey of a 
small central region of other more distant cluster, namely A2151 (Hercules).

Independently of this Spanish group, Vaduvescu (2005) studied the infrared properties of SF dwarf galaxies to trace the evolutionary link between dIs and BCDs, selecting two galaxy samples located in the Local Volume (LV, $d<10 \mathrm{Mpc})$ and Virgo cluster. Physical and chemical links between dIs and BCDs were found (Vaduvescu et al. 2005, 2006, 2007), suggesting strong links between these two classes. A new surface photometry law for dIs was proposed using the hyperbolic secant ("sech") which models both the exponential outer profile and the flat inner region, and another fit was proposed for BCDs using the same sech law to model the extended old component plus a Gaussian to mimic the younger central starburst. Using available HI velocity line-widths which characterise the kinetic energy, another result was the apparent discovery of a "dwarf fundamental plane" (FP) which links luminosity to rotation and could be used as a distance indicator for dIs and BCDs located in the nearby universe (Vaduvescu \& McCall 2008; McCall et al. 2012). During the last years, we extended this study to other three nearby clusters: Fornax, Hydra (Vaduvescu et al. 2011) and Antlia (Vaduvescu et al. 2014). Nevertheless, the main problem remains the lack of radio detection of the very faint dIs and BCDs, especially at further distances.

Light in visible is not the best quantity to trace mass in SF galaxies, mainly because younger stars overwhelm most of the old population. Instead, the near infrared (NIR) domain provides an opportunity (especially in the $K$ bands, redder than $J$ and less contaminated by the atmosphere which affects mostly $H$ ), being also more transparent through the Galactic and internal dust (up to about ten times more than in visible).

The present paper continues this past work of the authors, proposing to study physical and chemical properties of another sample of 45 SF dwarfs (mostly BCDs) located in four other clusters, namely A779, A1367, A1656 (Coma) and A2151 (Hercules) for which we collected deep NIR imaging during one observing run using the TNG telescope in 2010. Section 2 presents the cluster and galaxy sample, Sect. 3 the NIR imaging observations, and Sect. 4 the derived surface photometry. In Sect. 5 we derive galaxy stellar masses and we test correlations between physical and chemical parameters using metallicity and radio data from the literature. We discuss findings in Sect. 6 and draw the conclusions in Sect. 7.

\section{Sample}

\subsection{Cluster selection}

Building on our past work, we chose the following criteria for cluster selection:

1. location relatively close (distance $d<150 \mathrm{Mpc}$ ) at low redshift $(z<0.04)$

2. previously studied in $\mathrm{H} \alpha$ and via spectroscopy by the Granada group;

3. visible from La Palma during spring time during part of the granted nights.

Based on these criteria, we selected the following four clusters.

\subsubsection{Abell 779}

According to NED database, A779 is located at a galactocentric distance $91.9 \pm 7.3 \mathrm{Mpc}$ (distance modulus $(m-M)=$ $34.82 \mathrm{mag}$ ), receding with a heliocentric radial velocity $v=6742 \mathrm{~km} \mathrm{~s}^{-1}$ (redshift $z=0.022489$ ). It spans on sky $90^{\prime}$ in diameter and has a richness class 83 .
The cluster A779 has a low velocity dispersion (Hwang et al. 2008; Coziol et al. 2009), however it presents a significant potential well as defined by a nearly circular X-ray emission detected by ROSAT, with the well known cD galaxy NGC 2832 dominating the inner region of the cluster. The cluster is elongated in the north-south direction. A779 was surveyed in H-alpha by Reverte et al. (2007) and Reverte (2008) who encountered many SF galaxy candidates (120 at $5 \sigma$ ) from which for this run we selected nine targets.

\subsubsection{Abell 1367}

Located at a galactocentric distance $89.7 \pm 6.3 \mathrm{Mpc}((m-M)=$ $34.76 \mathrm{mag})$, the cluster A1367 has a velocity $v=6595 \mathrm{~km} \mathrm{~s}^{-1}$ (redshift $z=0.022$ ), spanning on sky 155' in diameter and having a population richness class 85 . A1367 is an excellent place to study the impact of the cluster environment on the transition from gas-rich to stellar dominated systems because it is a dynamically young cluster at the intersection of two Great Wall filaments (Cortese et al. 2008). Moreover, the Arecibo Galaxy Environment Survey has completed a HI survey of $5 \mathrm{deg}^{2}$ centred on the cluster, and Cortese et al. (2003) reports optical spectroscopy of 60 galaxies projected on the direction of A1367.

Kraiwattanawong et al. (2009) found that most galaxies with strong emission lines in A1367 appear disturbed, which makes this cluster an interesting sample to study environmental effects on star formation. A1356 was surveyed in $\mathrm{H} \alpha$ by Iglesias-Paramo et al. (2002) and Iglesias-Paramo et al. (2003) who studied its luminosity function in both $\mathrm{H} \alpha$ and $r$. Among the 41 galaxies detected in $\mathrm{H} \alpha$ by this work, we selected 12 targets for NIR imaging.

\subsubsection{Abell $1656=$ Coma}

Coma is a very studied galaxy cluster (1535 references on $\mathrm{NED}^{1}$, surpassing even Virgo). It is the richest cluster in our sample (richness class 484), being located at a distance of $95.1 \pm 6.7 \mathrm{Mpc}((m-M)=34.89 \mathrm{mag})$ and moving with a velocity $v=6925 \mathrm{~km} \mathrm{~s}^{-1}$ (redshift $z=0.023100$ ). It spans on sky more than $5^{\circ}$ in diameter, appearing quite close to Abell 1367 $\left(19^{\circ}\right.$ between cluster centres).

Iglesias-Paramo et al. (2002) mapped the $\mathrm{H} \alpha$ luminosity function in the centre of Coma (one square degree) and compared it with that of Virgo, finding the star-formation rate (SFR) contribution of Coma is about $0.25 \%$ of the SFR per unit volume of the local Universe. This work includes four targets included in the present paper.

Mahajan et al. (2010) used Spitzer $24 \mu \mathrm{m}$ and SDSS DR7 data to investigate the properties of SF galaxies residing in the central $2 \times 2 \mathrm{deg}^{2}$ of the Coma cluster, finding that star formation in dwarfs is quenched at the centre while passively evolving massive galaxies are found in all environments. They found that many of the blue galaxies in Coma are post-starburst galaxies, suggesting also that a significant fraction of the blue galaxies are currently on their first infall towards the cluster. In their next paper, Mahajan et al. (2011) employed SDSS DR7 data to study the evolution of dwarf galaxies located in a very large area covering $500 \mathrm{deg}^{2}$ in the Coma supercluster. The blue colour of some post-starburst dwarfs found within the Coma cluster virial radius suggests that the star formation was quenched very rapidly in the last 500 Myr. The authors identified two populations of blue dwarfs, suggesting that one represents the progenitors of the

\footnotetext{
1 https://ned.ipac.caltech.edu
} 
passive dwarf galaxies abundantly found in the cores of lowredshift rich clusters, such as Coma.

\subsubsection{Abell $2151=$ Hercules}

Hercules is the farthermost from our cluster sample, located at a galactocentric distance of $151.6 \pm 10.6 \mathrm{Mpc}((m-M)=35.90$ mag) and moving with a velocity $v=10972 \mathrm{~km} \mathrm{~s}^{-1}$ (redshift $z=0.036600$ ). Like Coma, it appears very extended, with a diameter of about $6^{\circ}$.

Iglesias-Paramo et al. (2003) selected a sample of 22 galaxies previously detected by a blind VLA HI survey of the Hercules cluster. In this paper we include six targets for which the mentioned paper provide HI velocity line-widths W20, oxygen abundances and deep imaging photometry in the visible. Surface brightness profiles (SBPs) in $B V I$ appear exponential in the outer part, but at the inner part (up to $\sim 5^{\prime \prime}$ ) they reveal an excess which levels off close to the centre, similar to a Gaussian component.

Continuing these efforts, Cedres et al. (2009) performed a deep $\mathrm{H} \alpha$ of the centre of the Hercules cluster (only about $0.2 \mathrm{deg}^{2}$ total), detecting 50 sources in $\mathrm{H} \alpha$ from which we selected 17 targets for our paper. Based on this sample, Petropoulou et al. (2011) acquired longslit spectroscopy for 27 SF galaxies, from which we include in this present paper nine dwarfs. Most Hercules SF galaxies follow typical $M Z$ and $L Z$ sequences, though the dwarf galaxies located at the densest regions appear to be outliers to these global relations, suggesting a physical reason.

In a recent WHT/AF2 spectroscopic work focused on the central 45' of Hercules, (Agulli et al. 2017) found that the cluster is still in collapsing phase. The evolution of dwarfs is driven by slow environmental processes in a hostile environment capable to remove the gas reservoirs and quench the star formation.

\subsection{Galaxy selection}

We used the following selection criteria to select our targets:

1. SF galaxies detected in our previous $\mathrm{H} \alpha$ surveys;

2. cluster membership, defined by either SDSS spectroscopic or photometric redshift consistent with cluster membership;

3 . located in relatively quiet X-ray regions of the cluster, to avoid complicated environmental influences;

4. dwarfs $M_{\mathrm{B}} \geq-18$ or $M_{\mathrm{r}} \geq-19$;

5. preferably detected via spectroscopy by the SDSS8 survey (available in 2010);

6. having more (preferably at least five) 2MASS stars in the small 4' field to reduce our NIR photometry.

Based on these criteria, we selected a total of 45 objects: 9 galaxies in A779, 12 objects in A1367, four targets in Coma and 20 galaxies in Hercules. In Fig. 1 we plot our targets overlaid on the DSS images, by adding the ROSAT X-ray contours from all sky surveys $0.1-2.4 \mathrm{keV}$.

\section{Observations}

Four nights were awarded at Roque de los Muchachos Observatory (ORM) by the Spanish TAC between 30 April and 3 May 2010 (programme number CAT_110). We used the Italian Telescopio Nationale Galileo (TNG) which is equipped at one Nasmyth focus with the Near Infrared Camera Spectrometer (NICS) based on a $\mathrm{HgCdTe}$ Hawaii $1024 \times 1024$ array (pixel size $0.25^{\prime \prime}$ used for imaging over a relatively small field (FOV) $4.2^{\prime} \times 4.2^{\prime}$. We used $K^{\prime}$ band for all our targets. The weather was

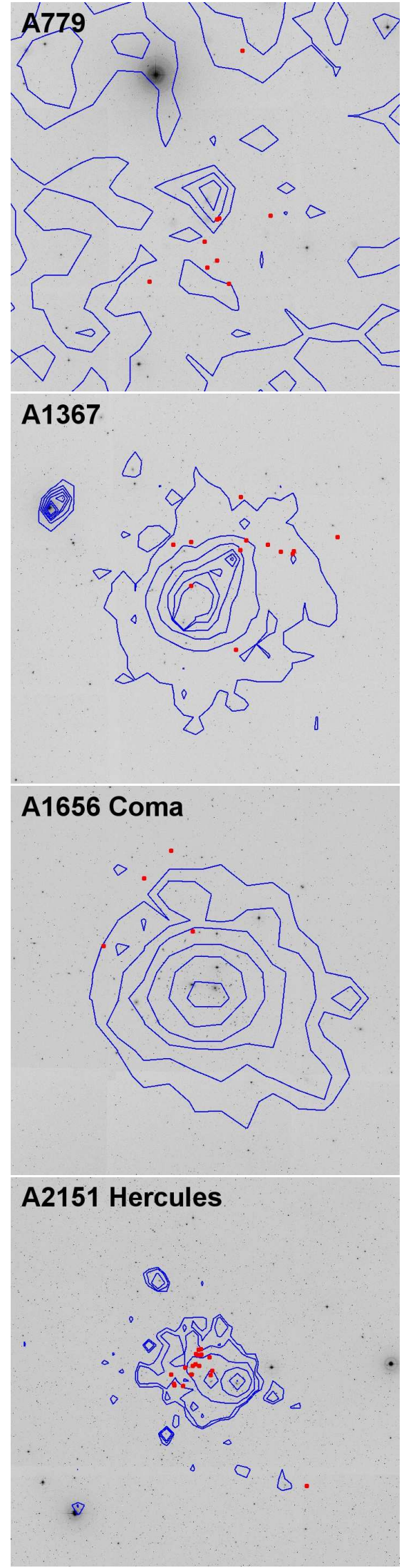

Fig. 1. Dwarf galaxies observed in the clusters A779, A1367, A1656 (Coma), and A2151 (Hercules) with the TNG. We mark the targets with red squares overlaid on the DSS images and the ROSAT X-ray contours. The field is about two degrees and the sky orientation is normal $(\mathrm{N}$ up, E left). 
Table 1. Star forming dwarf galaxies observed with the TNG in A779, A1367, Coma, and Hercules clusters.

\begin{tabular}{|c|c|c|c|c|c|c|c|c|c|}
\hline Galaxy & Short name & Obs. date & Exp & $m_{r}$ & $\log M_{\mathrm{HI}}$ & $12+\log (\mathrm{O} / \mathrm{H})$ & $\log L(\mathrm{H} \alpha)$ & $W_{20}$ & Dist \\
\hline \multicolumn{10}{|c|}{ Abell 779} \\
\hline J091810.99+333955.3 & 779-9553 & 20100502 & 1500 & 17.67 & - & 8.19 & 40.09 & - & 101.8 \\
\hline J091852.59+343056.6 & 779-0566 & 20100502 & 1380 & 16.60 & - & - & 39.98 & - & 89.3 \\
\hline J091913.04+331856.2 & $779-8562$ & 20100502 & 1500 & 17.22 & - & 8.53 & 39.60 & - & 91.8 \\
\hline J091931.13+333852.0 & $779-8520$ & 20100502 & 1800 & 16.43 & - & 8.06 & 40.78 & - & 96.8 \\
\hline J091928.07+333909.2 & 779-9092 & 20100502 & 1800 & 17.47 & - & - & 39.95 & - & 96.7 \\
\hline J091931.26+332603.8 & $779-6038$ & 20100503 & 1800 & 17.15 & - & 8.37 & 40.21 & - & 92.7 \\
\hline J091945.40+332357.1 & $779-3571$ & 20100503 & 1500 & 17.78 & - & 8.41 & 39.84 & - & 97.9 \\
\hline J091949.10+333159.6 & $779-1596$ & 20100503 & 1800 & 17.45 & - & 8.19 & 40.23 & - & 87.6 \\
\hline J092111.29+331932.6 & 779-9326 & 20100502 & 1500 & 17.25 & - & - & 40.45 & - & 129.4 \\
\hline \multicolumn{10}{|c|}{ Abell 1367} \\
\hline $\mathrm{J} 114141.22+200231.9$ & $1367-2319$ & 20100430 & 1500 & 17.45 & - & 8.13 & 39.94 & - & 115.0 \\
\hline $\mathrm{J} 114239.31+195808.1$ & $1367-8081$ & 20100501 & 1800 & 17.09 & - & 8.36 & 40.19 & - & 99.9 \\
\hline J114240.34+195717.0 & $1367-7170$ & 20100501 & 1800 & 17.86 & - & - & 39.40 & - & 102.1 \\
\hline J114256.45+195758.3 & $1367-7583$ & 20100502 & 1560 & 15.53 & 9.97 & 8.50 & 41.26 & - & 99.0 \\
\hline $\mathrm{J} 114313.09+200017.3$ & $1367-0173$ & 20100502 & 1800 & 15.57 & 9.94 & 8.38 & 41.35 & - & 95.4 \\
\hline J114341.54+200137.0 & $1367-1370$ & 20100501 & 1800 & 17.20 & - & 8.48 & 39.82 & - & 87.7 \\
\hline $\mathrm{J} 114348.89+201454.0$ & $1367-4540$ & 20100430 & 1500 & 16.01 & - & 8.55 & 40.96 & - & 82.9 \\
\hline $\mathrm{J} 114349.87+195834.8$ & $1367-8348$ & 20100501 & 1320 & 16.46 & - & - & 40.11 & - & 102.6 \\
\hline J114355.78+192746.1 & $1367-7461$ & 20100501 & 1500 & 18.82 & - & - & 39.29 & - & 87.3 \\
\hline $\mathrm{J} 114454.61+194732.5$ & $1367-7325$ & 20100501 & 1800 & 19.98 & - & - & 40.17 & - & 109.8 \\
\hline $\mathrm{J} 114454.56+200101.4$ & $1367-1014$ & 20100430 & 1500 & 16.26 & - & 8.58 & 39.66 & - & 99.2 \\
\hline $\mathrm{J} 114518.13+200009.0$ & $1367-0090$ & 20100501 & 1800 & 17.47 & - & - & 39.51 & - & 72.3 \\
\hline \multicolumn{10}{|c|}{ Abell $1656=$ Coma } \\
\hline $\mathrm{J} 130006.25+281500.5$ & $1656-5005$ & 20100502 & 1800 & 18.41 & - & - & 39.55 & - & $(95.1)$ \\
\hline $\mathrm{J} 130037.14+283950.8$ & $1656-9508$ & 20100502 & 1380 & 16.93 & - & 8.52 & 39.41 & - & 97.2 \\
\hline $\mathrm{J} 130114.95+283118.3$ & $1656-1183$ & 20100502 & 1500 & 17.13 & - & 8.30 & 40.16 & - & 115.8 \\
\hline $\mathrm{J} 130211.94+281023.6$ & $1656-0236$ & 20100502 & 1800 & 16.41 & - & 8.16 & 40.62 & - & $(95.1)$ \\
\hline \multicolumn{10}{|c|}{ Abell $2151=$ Hercules } \\
\hline $\mathrm{J} 160304.20+171126.7$ & $2151-1267$ & 20100501 & 1800 & 17.88 & - & 8.41 & 40.42 & - & 149.7 \\
\hline $\mathrm{J} 160506.81+174701.9$ & $2151-7019$ & 20100430 & 1500 & 17.85 & 8.95 & 8.27 & 40.01 & 189 & 137.1 \\
\hline J160508.79+174545.1 & $2151-5451$ & 20100430 & 1500 & 20.48 & - & - & 39.67 & - & $(151.6)$ \\
\hline $\mathrm{J} 160510.46+175116.0$ & $2151-1160$ & 20100430 & 1500 & 17.08 & 9.53 & 8.35 & 40.29 & 183 & 136.2 \\
\hline J160520.66+175201.4 & $2151-2014$ & 20100430 & 1500 & 18.51 & - & - & 39.80 & - & $(151.6)$ \\
\hline $\mathrm{J} 160521.73+175156.2$ & $2151-1562$ & 20100430 & 1500 & 17.80 & 8.87 & 8.26 & 39.63 & 101 & 160.1 \\
\hline $\mathrm{J} 160521.50+175337.8$ & $2151-3378$ & 20100430 & 1500 & 19.55 & - & - & 39.00 & - & $(151.6)$ \\
\hline $\mathrm{J} 160524.27+175329.3$ & $2151-3293$ & 20100501 & 1560 & 19.82 & - & 7.99 & 39.53 & - & 153.4 \\
\hline $\mathrm{J} 160524.99+175150.5$ & $2151-1505$ & 20100501 & 1560 & 18.04 & - & 8.56 & 39.86 & - & 142.5 \\
\hline $\mathrm{J} 160528.22+175212.2$ & $2151-2122$ & 20100501 & 1560 & 19.72 & - & - & 39.28 & - & $(151.6)$ \\
\hline $\mathrm{J} 160523.66+174832.3$ & $2151-8323$ & 20100501 & 1800 & 18.66 & - & 8.41 & 39.84 & - & 142.9 \\
\hline $\mathrm{J} 160528.84+174906.2$ & 2151-9062 & 20100501 & 1800 & 18.19 & - & - & 39.69 & - & $(151.6)$ \\
\hline J160531.84+174826.1 & $2151-8261$ & 20100430 & 1560 & 17.88 & - & 8.65 & 39.82 & - & 131.3 \\
\hline $\mathrm{J} 160533.36+174548.2$ & $2151-5482$ & 20100430 & 1560 & 18.76 & - & - & 39.82 & - & $(151.6)$ \\
\hline $\mathrm{J} 160534.05+174546.7$ & $2151-5467$ & 20100430 & 1560 & 24.03 & - & - & 39.44 & - & (151.6) \\
\hline $\mathrm{J} 160541.92+174758.3$ & $2151-7583$ & 20100430 & 1560 & 18.42 & 10.27 & 8.36 & 40.71 & 666 & 140.9 \\
\hline $\mathrm{J} 160544.81+174220.1$ & $2151-2201$ & 20100430 & 1500 & 20.27 & 9.34 & - & 38.35 & 150 & 153.4 \\
\hline $\mathrm{J} 160556.00+174233.9$ & $2151-2339$ & 20100430 & 1500 & 19.30 & 9.28 & 7.58 & 39.83 & 211 & 154.0 \\
\hline J160556.98+174304.1 & $2151-3041$ & 20100430 & 1500 & 18.53 & - & 8.24 & 40.15 & - & 160.5 \\
\hline J160600.14+174551.9 & $2151-5519$ & 20100501 & 1500 & 18.02 & 9.56 & 8.07 & 39.56 & - & 165.6 \\
\hline
\end{tabular}

Notes. Besides the TNG observing log, we include some data from the literature used in this paper.

clear during the first three nights with typical seeing $\sim 0.5^{\prime \prime}$, but became cloudy during the fourth night when we needed to close after only $2 \mathrm{~h}$. The Moon was bright (after Full Moon), known to affect less $K^{\prime}$ observations.

We exposed between 22 and 30 min all targets, using 1 min exposures (ndit $=4$ on-chip $15 \mathrm{~s}$ ) and cycling the small targets in the four quadrants of the chip, so that we could sample the sky background from the previous image.

Table 1 includes some published data regarding the TNG observed galaxies ordered for each clusters in right ascension. We list the following columns: the SDSS DR14 galaxy name (which includes the $\alpha$ and $\delta \mathrm{J} 2000$ coordinates), our short 
name (formed by cluster number plus last four numbers), the observation date (start of TNG night), total exposure time in $K^{\prime}$ (in seconds), the SDSS DR12 $r$-band apparent magnitude, the logarithm of the HI gas mass in $10^{9}$ solar masses (Iglesias-Paramo et al. 2003) and (Petropoulou 2012), the oxygen abundance (Petropoulou 2012), logarithm of $\mathrm{H} \alpha$ luminosity $\left(10^{-15} \mathrm{erg} \mathrm{s}^{-1}\right)$ calculated based on the $\mathrm{H} \alpha+\mathrm{N}$ fluxes (Reverte 2008; Cedres et al. 2009), the HI velocity line-width at $20 \%$ high (Iglesias-Paramo et al. 2003), and the distance in Mpc based on the Hubble flow distance (NED) or cluster distance in parenthesis where velocity information was not available.

\section{Surface photometry}

The contribution of the sky (especially affected by the bright nearby Moon) and of the environment (telescope optics and dome) could affect NIR imaging due to rapid variability in both time and space, and this becomes very important when observing very faint dwarf galaxies (Vaduvescu \& McCall 2004). We adopted a prudent approach, by subtracting the background taken from the previous science exposure in the nearby dithered position.

The NIR image reduction was performed in IRAF using a script developed in house. The script subtracts the sky observed in the previous image from the current galaxy image, then aligns and combines all individual exposures in the final galaxy image. For each galaxy field we derived the photometric $K^{\prime}$ zero points based on a few (about 5) 2MASS stars available, resulting in zero point errors typically less than $0.1 \mathrm{mag}$. We present the reduced $K^{\prime}$ images in the left panel of Fig. A.1, ordered by clusters and right ascension.

Following our past work (Vaduvescu et al. 2006, 2011, 2014) one could model galaxy profiles of SF dwarfs (BCD candidates) with a sech law to account for the extended light associated with the older stellar population plus a Gaussian law to fit the inner starburst. Accordingly, the sech and Gaussian surface brightness components $\mu_{\mathrm{S}}$ and $\mu_{\mathrm{G}}$ at radius $r$ are expressed as:

$\mu_{\mathrm{S}}=\mu_{0 \mathrm{~S}}-2.5 \log \left(\operatorname{sech}\left(\frac{r}{r_{0 \mathrm{~S}}}\right)\right)$

and

$\mu_{\mathrm{G}}=\mu_{0 \mathrm{G}}-2.5 \log \left(\exp \left(-\frac{1}{2}\left(\frac{r}{r_{0 \mathrm{G}}}\right)^{2}\right)\right)$.

Here the central surface brightness $\mu_{0 \mathrm{~S}}$ and the sech scale length $r_{0 \mathrm{~S}}$ characterise the outer component of the galaxy fitted by the sech law (bearing the "S" subscript), while the central surface brightness $\mu_{0 \mathrm{G}}$ and the Gaussian scale length $r_{0 \mathrm{G}}$ model the central outburst using the Gaussian fit (bearing the " $G$ " subscript).

Using these two components, the total surface brightness $\mu$ at radius $r$ can be expressed as:

$\mu=\mu_{\mathrm{O}}-2.5 \log \left(I_{0 \mathrm{~S}} \operatorname{sech}\left(\frac{r}{r_{0 \mathrm{~S}}}\right)+I_{0 \mathrm{G}} \exp \left(-\frac{1}{2}\left(\frac{r}{r_{0 \mathrm{G}}}\right)^{2}\right)\right)$,

where $z p$ is the "surface zero-point" derived from a few 2MASS stars identified in each field using the pixel scale of the instrument, and $I_{0 \mathrm{~S}}$ and $I_{0 \mathrm{G}}$ represent the central intensities associated with central surface brightness $\mu_{0 \mathrm{~S}}$ and $\mu_{0 \mathrm{G}}$, respectively.

The sech and Gaussian magnitude components $m_{\mathrm{S}}$ and $m_{\mathrm{G}}$ are simply expressed as a function of the total flux of the two components $I_{\mathrm{S}}$ and $I_{\mathrm{G}}$ as:

$m_{\mathrm{S}}=z p-2.5 \log \left(I_{\mathrm{S}}\right)$ and

$m_{\mathrm{G}}=z p-2.5 \log \left(I_{\mathrm{G}}\right)$.

In the right panels of Fig. A.1 we plot with round symbols the $K^{\prime}$ surface brightness profiles of all targets together with uncertainties. The sech component is overlaid with a thick solid line, the Gaussian component with a dashed line, and the sum of these two with a thin line which matches very well most of the observed surface brightness profiles.

Table 2 includes the following data derived in the $K^{\prime}$ band in our TNG run: galaxy short name (following the same order in Table 1), the ellipticity and position angle, the total (asymptotic) apparent magnitude $\left(m_{\mathrm{TK}}\right)$, the sech apparent magnitude $\left(m_{\mathrm{SK}}\right)$, the central surface brightness of the sech fit ( $\mu_{0 \mathrm{~S}}$, in $\mathrm{mag} / \mathrm{sq}$ arcsec) the apparent scale length of the sech fit $\left(r_{0 \mathrm{~S}}^{\prime \prime}\right.$ in $\left.\operatorname{arcsec}\right)$, the semimajor axis at surface brightness $K^{\prime}=22 \mathrm{mag} / \mathrm{sq}$ arcsec of the sech fit $\left(r_{22}^{\prime \prime}\right.$, in $\left.\operatorname{arcsec}\right)$, the physical scale length of the sech fit ( $r_{0 \mathrm{~S}}$ in $\left.\mathrm{kpc}\right)$, the physical semimajor axis at $r_{22}\left(r_{22}\right.$ in kpc), the colour $r-K^{\prime}$ (based on the SDSS $m_{r}$ from Table 1$)$, the sech absolute magnitude $\left(M_{\mathrm{SK}}\right)$, the total (asymptotic) absolute magnitude $M_{\mathrm{TK}}$, and the logarithm of the stellar mass of the sech component $\left(\log M_{\mathrm{SK}}^{*}\right)$ calculated according to Vaduvescu et al.(2007, Eq. (8)). A few targets have no radial velocity data, in which case we calculated their distance scale and physical radii by assuming average cluster scales taken from NED; we include these values in parentheses.

Eight galaxies (seven in A779 and one in Coma) have been covered by the UKIDSS Large Area Survey (LAS) using short exposures $4 \times 10 \mathrm{~s}$ (Lawrence et al. 2007) and other three have been detected by 2MASS survey (in A1367) using very short exposures of $8 \mathrm{~s}$ (Kleinmann et al. 1994). The UKIDSS DR9 Petrosian magnitudes are in average 0.35 mag fainter than our TNG total magnitudes $\left(m_{\mathrm{TK}}\right)$, while the 2MASS extended magnitudes are only 0.04 mag shallower than our TNG measurements.

\section{Correlations}

We study SF dwarfs in clusters by comparing our previous data in the Local Volume (LV - plotted with black solid circles) and nearby clusters Virgo, Fornax, Hydra, and Antlia (acronym VFHA, plotted with black open circles) with data presented in this paper in Abell 779 (red triangles), Abell 1367 (blue stars), Coma (magenta crosses) and Hercules clusters (green squares).

\subsection{Physical correlations}

Figure 2 plots the semimajor axis $r_{22 S K}$ in kpc (Vaduvescu et al. 2005) as a function of the sech absolute magnitude $M_{\mathrm{SK}}$, both calculated based on the Hubble flow distance given by NED. The previously known linear correlation in the LV and the four nearby clusters is plotted as a dashed line (Vaduvescu et al. 2006). The growing trend is probed by the four distant Abell clusters but the linear trend breaks above $M_{\mathrm{SK}}=-19$, with brightest SF dwarfs up to three times larger than expected from the linear fit. 2151-5519 (LEDA 140568, classified as S-type in NED) is extremely faint and clearly shows two entities in the SDSS image separated by about $5^{\prime \prime}$. In the TNG image they appear linked and were included in the fit, resulting in a larger size (possibly double than real) for this target.

Figure 3 shows the sech scale length $r_{0 \mathrm{SK}}$ as a function of the sech absolute magnitude $M_{\mathrm{SK}}$. The previous correlation for dwarfs in the local universe is plotted with a dashed line. The growing trend is continued by members of more distant 
Table 2. Physical parameters of the galaxies observed in A779, A1367, Coma, and Hercules clusters derived in this paper.

\begin{tabular}{|c|c|c|c|c|c|c|c|c|c|c|c|c|c|}
\hline Short name & $e$ & $P A$ & $m_{\mathrm{TK}}$ & $m_{\mathrm{SK}}$ & $\mu_{0 \mathrm{~S}}$ & $r_{0 \mathrm{~S}}^{\prime \prime}$ & $r_{22}^{\prime \prime}$ & $r_{0 S}$ & $r_{22}$ & $r-K^{\prime}$ & $M_{\mathrm{SK}}$ & $M_{\mathrm{TK}}$ & $\log M_{\mathrm{SK}}^{*}$ \\
\hline \multicolumn{14}{|c|}{ Abell 779} \\
\hline 779-9553 & 0.4 & +80 & 15.70 & 15.84 & 19.64 & 2.2 & 6.3 & 1.1 & 3.1 & 1.97 & -19.19 & -19.34 & 8.90 \\
\hline 779-0566 & 0.4 & -70 & 14.17 & 13.99 & 19.90 & 5.8 & 15.1 & 2.5 & 6.5 & 2.43 & -20.78 & -20.58 & 9.53 \\
\hline 779-8562 & 0.6 & +30 & 14.73 & 14.84 & 18.37 & 2.4 & 9.5 & 1.1 & 4.2 & 2.49 & -19.97 & -20.08 & 9.21 \\
\hline $779-8520$ & 0.2 & +10 & 14.07 & 14.60 & 16.76 & 0.9 & 4.9 & 0.4 & 2.3 & 2.36 & -20.33 & -20.86 & 9.35 \\
\hline 779-9092 & 0.4 & -45 & 15.58 & 16.00 & 18.54 & 1.2 & 4.8 & 0.6 & 2.3 & 1.89 & -18.93 & -19.35 & 8.79 \\
\hline 779-6038 & 0.6 & +90 & 14.78 & 15.38 & 17.18 & 1.1 & 5.5 & 0.5 & 2.5 & 2.37 & -19.46 & -20.06 & 9.01 \\
\hline $779-3571$ & 0.2 & -40 & 15.24 & 15.44 & 18.40 & 1.3 & 5.2 & 0.6 & 2.5 & 2.54 & -19.52 & -19.71 & 9.03 \\
\hline 779-1596 & 0.4 & -5 & 15.15 & 15.61 & 18.61 & 1.5 & 5.8 & 0.6 & 2.5 & 2.30 & -19.10 & -19.56 & 8.86 \\
\hline 779-9326 & 0.4 & -80 & 15.09 & 15.06 & 20.00 & 3.7 & 9.3 & 2.3 & 5.8 & 2.16 & -20.50 & -20.47 & 9.42 \\
\hline \multicolumn{14}{|c|}{ Abell 1367} \\
\hline $1367-2319$ & 0.4 & -50 & 15.28 & 17.05 & 18.77 & 0.8 & 3.1 & 0.4 & 1.7 & 2.17 & -18.26 & -20.02 & 8.53 \\
\hline $1367-8081$ & 0.4 & +20 & 14.93 & 14.97 & 18.91 & 2.3 & 8.3 & 1.1 & 4.0 & 2.16 & -20.03 & -20.07 & 9.23 \\
\hline $1367-7170$ & 0.4 & +60 & 15.37 & 15.50 & 20.25 & 3.4 & 7.8 & 1.7 & 3.9 & 2.49 & -19.55 & -19.68 & 9.04 \\
\hline $1367-7583$ & 0.0 & 0 & 12.97 & 12.98 & 18.98 & 4.8 & 16.7 & 2.3 & 8.0 & 2.56 & -22.00 & -22.01 & 10.02 \\
\hline $1367-0173$ & 0.6 & -75 & 12.99 & 13.11 & 18.63 & 5.9 & 22.5 & 2.7 & 10.4 & 2.58 & -21.79 & -21.91 & 9.94 \\
\hline $1367-1370$ & 0.7 & -20 & 15.04 & 16.10 & 18.30 & 1.5 & 6.1 & 0.6 & 2.6 & 2.16 & -18.62 & -19.67 & 8.67 \\
\hline $1367-4540$ & 0.2 & -10 & 13.65 & 14.23 & 17.77 & 1.7 & 7.7 & 0.7 & 3.1 & 2.36 & -20.36 & -20.94 & 9.37 \\
\hline $1367-8348$ & 0.6 & +60 & 13.58 & 13.77 & 16.07 & 1.3 & 8.3 & 0.6 & 4.1 & 2.88 & -21.29 & -21.48 & 9.74 \\
\hline $1367-7461$ & 0.3 & +40 & 16.47 & 16.34 & 21.37 & 3.6 & 4.2 & 1.5 & 1.8 & 2.35 & -18.36 & -18.24 & 8.57 \\
\hline $1367-7325$ & 0.2 & +90 & 16.49 & 16.96 & 18.98 & 0.8 & 2.9 & 0.4 & 1.5 & 3.49 & -18.24 & -18.71 & 8.52 \\
\hline $1367-1014$ & 0.1 & -80 & 13.76 & 15.23 & 18.03 & 1.1 & 4.9 & 0.5 & 2.4 & 2.50 & -19.75 & -21.22 & 9.12 \\
\hline $1367-0090$ & 0.4 & +60 & 15.37 & 15.60 & 20.34 & 3.4 & 7.4 & 1.2 & 2.6 & 2.10 & -18.69 & -18.93 & 8.70 \\
\hline \multicolumn{14}{|c|}{ Abell $1656=$ Coma } \\
\hline $1656-5005$ & 0.7 & -5 & & 16.16 & & 1.4 & 5.8 & $(0.6)$ & $(2.7)$ & 3.54 & -18.74 & -20.02 & 8.72 \\
\hline $1656-9508$ & 0.4 & +25 & 14.26 & 15.14 & 17.32 & 1.0 & 5.2 & 0.5 & 2.4 & 2.67 & -19.80 & -20.68 & 9.14 \\
\hline $1656-1183$ & 0.4 & +60 & 14.83 & 14.80 & 20.04 & 4.3 & 10.6 & 2.4 & 6.0 & 2.30 & -20.52 & -20.49 & 9.43 \\
\hline $1656-0236$ & 0.3 & +30 & 13.46 & 13.37 & 19.54 & 6.0 & 17.8 & $(2.8)$ & $(8.2)$ & 2.95 & -21.52 & -21.43 & 9.83 \\
\hline \multicolumn{14}{|c|}{ Abell $2151=$ Hercules } \\
\hline $2151-1267$ & 0.8 & +80 & 14.29 & 14.27 & 17.32 & 2.7 & 13.4 & 2.0 & 9.7 & 3.59 & -21.61 & -21.59 & 9.87 \\
\hline 2151-7019 & 0.2 & +50 & 16.00 & 16.05 & 19.68 & 1.8 & 5.0 & 1.2 & 3.3 & 1.85 & -19.64 & -19.69 & 9.08 \\
\hline $2151-5451$ & 0.1 & +10 & 17.17 & 17.02 & 19.68 & 1.1 & 3.0 & $(0.8)$ & $(2.2)$ & 3.31 & -18.88 & -18.73 & 8.78 \\
\hline $2151-1160$ & 0.5 & -40 & 14.83 & 14.72 & 20.41 & 5.7 & 12.3 & 3.8 & 8.1 & 2.25 & -20.95 & -20.84 & 9.60 \\
\hline 2151-2014 & 0.3 & +50 & 16.03 & 16.12 & 20.40 & 2.5 & 5.4 & $(1.8)$ & $(4.0)$ & 2.48 & -19.78 & -19.87 & 9.14 \\
\hline $2151-1562$ & 0.2 & -50 & 15.26 & 15.40 & 18.47 & 1.4 & 5.3 & 1.1 & 4.1 & 2.54 & -20.62 & -20.76 & 9.47 \\
\hline 2151-3378 & 0.2 & -20 & 16.83 & 16.68 & 21.46 & 3.0 & 3.2 & $(2.2)$ & $(2.4)$ & 2.72 & -19.22 & -19.07 & 8.91 \\
\hline $2151-3293$ & 0.1 & +90 & 17.68 & 18.01 & 18.79 & 0.4 & 1.6 & 0.3 & 1.2 & 2.14 & -17.92 & -18.25 & 8.39 \\
\hline $2151-1505$ & 0.5 & -40 & 15.73 & 15.80 & 20.28 & 3.3 & 7.4 & 2.3 & 5.1 & 2.31 & -19.97 & -20.04 & 9.21 \\
\hline $2151-2122$ & 0.3 & +30 & 16.32 & 16.84 & 17.82 & 0.6 & 2.5 & $(0.4)$ & $(1.8)$ & 3.40 & -19.06 & -19.58 & 8.85 \\
\hline $2151-8323$ & 0.6 & -40 & 15.81 & 15.93 & 19.97 & 3.0 & 7.6 & 2.1 & 5.3 & 2.85 & -19.84 & -19.84 & 9.16 \\
\hline 2151-9062 & 0.6 & -40 & 15.94 & 16.93 & 18.09 & 0.8 & 3.4 & $(0.6)$ & $(2.5)$ & 2.25 & -18.98 & -20.09 & 8.81 \\
\hline $2151-8261$ & 0.5 & -20 & 14.85 & 15.06 & 16.79 & 0.9 & 5.1 & 0.6 & 3.2 & 3.03 & -20.53 & -20.74 & 9.43 \\
\hline $2151-5482$ & 0.2 & -30 & 16.24 & 16.45 & 18.08 & 0.7 & 3.0 & $(0.5)$ & $(2.2)$ & 2.52 & -19.45 & -19.66 & 9.00 \\
\hline $2151-5467$ & 0.4 & +45 & 16.01 & 16.41 & 18.99 & 1.2 & 4.3 & $(0.9)$ & $(3.2)$ & 8.02 & -19.49 & -19.89 & 9.02 \\
\hline $2151-7583$ & 0.5 & -80 & 16.78 & 16.71 & 20.82 & 2.8 & 4.8 & 1.9 & 3.3 & 1.64 & -19.03 & -18.96 & 8.74 \\
\hline $2151-2201$ & 0.5 & +15 & 16.78 & 16.38 & 21.14 & 3.7 & 5.3 & 2.8 & 3.9 & 3.49 & -19.55 & -19.15 & 9.04 \\
\hline 2151-2339 & 0.1 & 0 & 17.46 & 17.56 & 19.81 & 0.9 & 2.4 & 0.7 & 1.8 & 1.84 & -18.38 & -18.48 & 8.57 \\
\hline $2151-3041$ & 0.6 & -75 & 16.91 & 16.80 & 19.25 & 1.4 & 4.7 & 1.1 & 3.7 & 1.62 & -19.23 & -19.12 & 8.92 \\
\hline 2151-5519 & 0.8 & +5 & 16.55 & 15.86 & 20.70 & 6.1 & 11.4 & 4.9 & 9.2 & 1.47 & -20.24 & -19.55 & 9.32 \\
\hline
\end{tabular}

Notes. Please refer to the Sect. 4 for the meaning of each column. The typical error bars are plotted in Figs. 2-12, being evaluated in Sect. 6.2.

clusters, but again the previous linear trend is broken and the cores vary up to five times in size. Again, 2151-5519 could be actually smaller, while 2151-1160 (classified as $S c$ in NED) actually resembles with a spiral in the SDSS image surrounded by a few blue clumps not detected in the TNG image.
Figure 4 represents the sech central surface brightness $\mu_{0 \mathrm{SK}}$ as a function of the sech absolute magnitude $M_{\mathrm{SK}}$. The dashed line represents the fit to most dIs and BCDs in the LV and Virgo cluster (Eq. (14) and Fig. 6 from Vaduvescu et al. 2006). This linear correlation does not hold for BCDs in the Abell clusters 


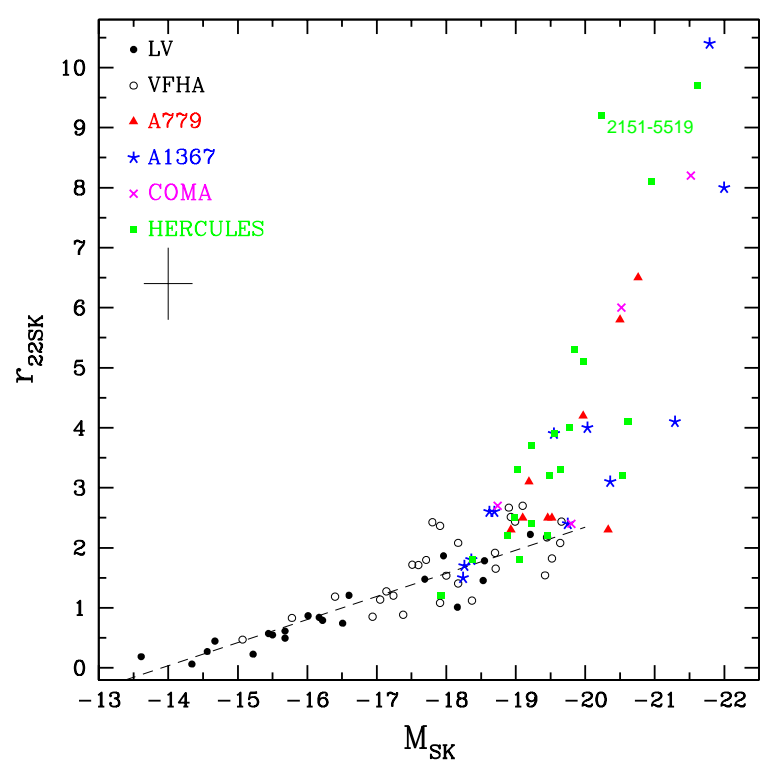

Fig. 2. Semimajor axis $r_{22 S K}$ (in kpc) of the star forming (SF) dwarf galaxies studied before in the Local Volume (LV), Virgo, Fornax, Hydra, Antlia (denoted jointly by VFHA, based on our previous work), and the four Abell clusters observed with the TNG (this paper) as a function of the luminosity - sech absolute magnitude $M_{\mathrm{SK}}$. The cross in the upper left shows typical uncertainties in the two parameters.

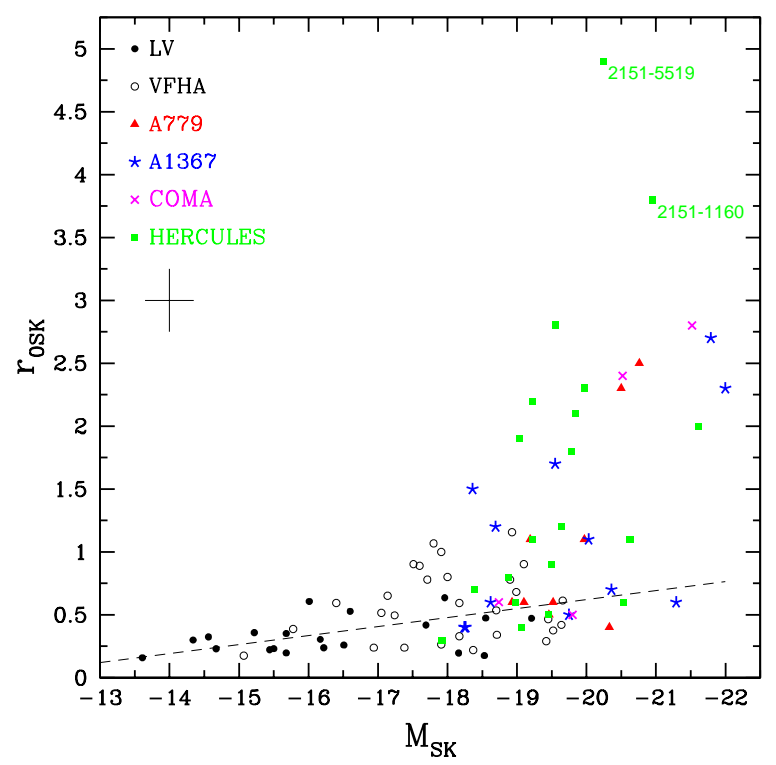

Fig. 3. Sech scale length $r_{0 \mathrm{SK}}$ (in kpc) of the SF dwarfs as a function of the sech absolute magnitude $M_{\mathrm{SK}}$. The previously known linear trend is marked with a dash line, but is broken for more luminous objects whose cores vary up to five times in size. The cross in the upper left shows typical uncertainties in the two parameters.

which have fainter sech cores (up to $\sim 2 \mathrm{mag} / \mathrm{sq} \operatorname{arcsec}$ ). Most galaxies in A1367, Hercules and Coma show fainter cores compared to galaxies in other clusters with similar $M_{\mathrm{SK}}$, and the environmental influences could be the origin of these differences, taking into account the compactness of these clusters visible in the X-ray maps presented in Fig. 1.

Figure 5 plots the $r-K^{\prime}$ colour of the targets observed with the TNG ( $K^{\prime}$ total asymptotic magnitude) having known Sloan $r$ model magnitudes in SDSS DR12. The colours range between

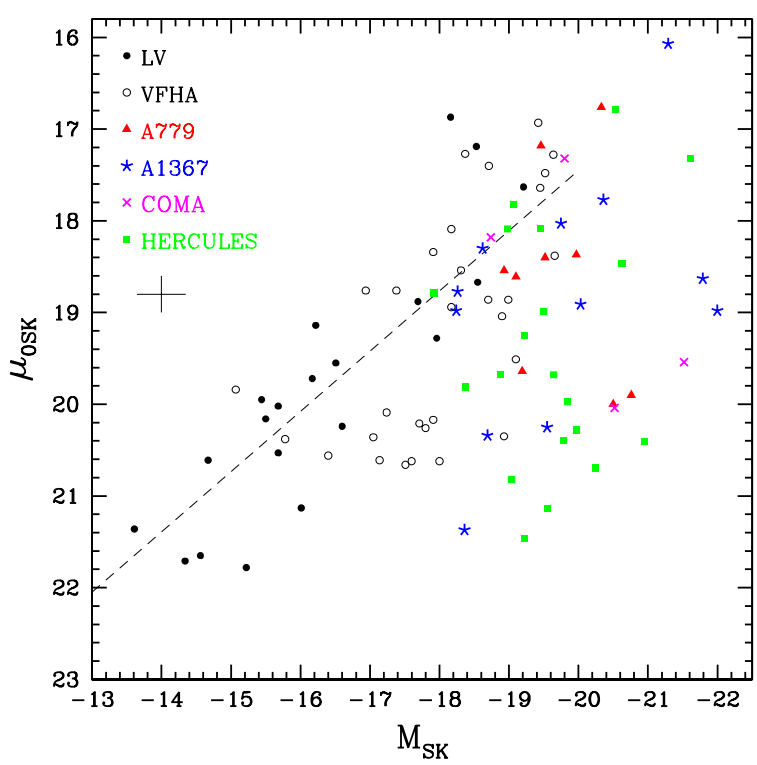

Fig. 4. Sech central surface brightness $\mu_{0 \mathrm{SK}}$ of the SF dwarfs as a function of sech absolute magnitude $M_{\mathrm{SK}}$. The dashed line represents the fit of most dIs and BCDs in the LV and Virgo cluster (Vaduvescu et al. 2006). Most galaxies in A1367, Hercules and Coma show fainter cores compared to galaxies in other clusters with similar luminosity, and the environmental influences could be the origin of these differences. The cross in the upper left shows typical uncertainties in the two parameters.

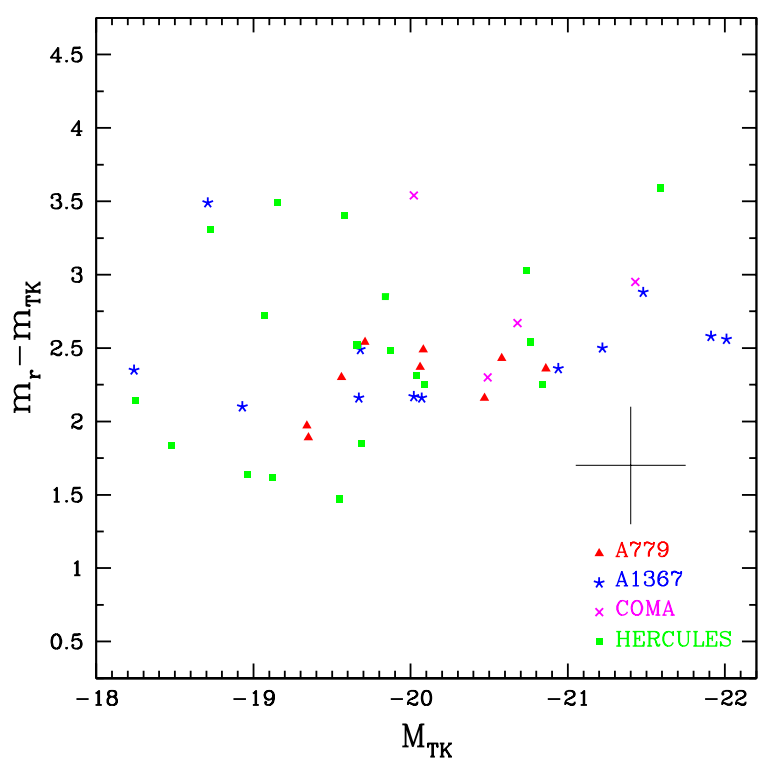

Fig. 5. Colour $r-K^{\prime}$ of the SF dwarfs as a function of total absolute magnitude $M_{\mathrm{TK}}$, with Sloan $r$ model magnitudes taken from SDSS DR12. The colours range between 1.5 and 3.5 mag and have a larger spread for fainter targets, probably due to larger uncertainties in the measurements. The cross in the bottom right shows typical uncertainties in the two parameters.

1.5 and 3.5 mag and have a larger spread for fainter targets, most probably due to larger uncertainties in the measurements.

Figure 6 shows the flux excess of the TNG Abell targets, expressed as the difference between the sech and the total magnitudes. Most of the galaxies are spread between -0.5 and +0.5 mag suggesting good sech fits for most SBPs, while a few galaxies have larger Gaussian components, between +0.5 and about +1.5 mag. Once again, the spread is larger for fainter objects. 


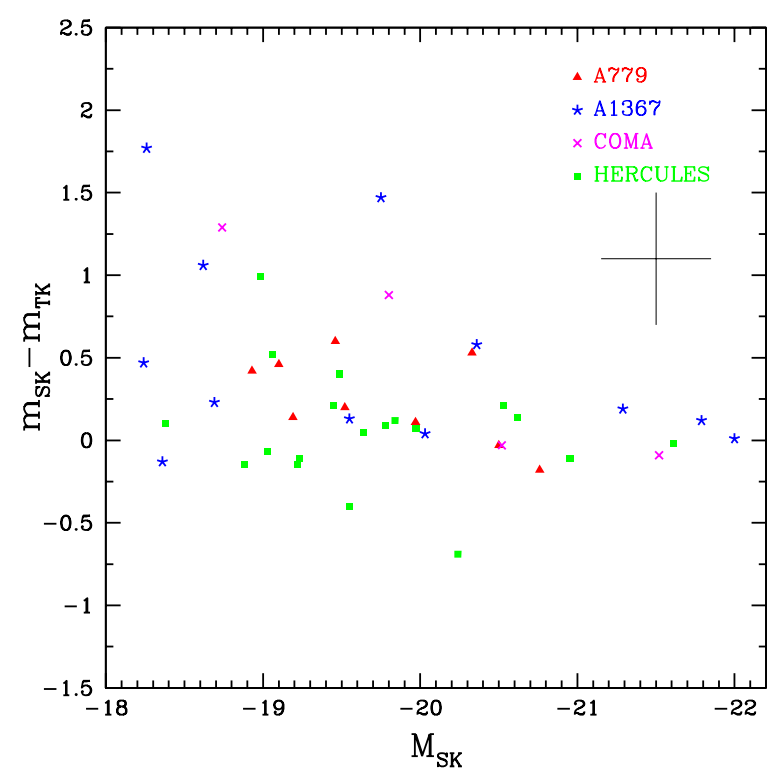

Fig. 6. Starburst excess, expressed as the difference sech minus total apparent magnitude $\left(m_{\mathrm{SK}}-m_{\mathrm{TK}}\right)$ in function of their luminosity (sech absolute magnitude $M_{\mathrm{SK}}$ ). Most galaxies are spread between -0.5 and +0.5 mag suggesting good sech fits, while a few others have larger Gaussian components, suggesting more intensive star forming activity. The cross in the upper right shows typical uncertainties in the two parameters.

Figure 7 represents the dwarf FP defined by 50 dIs (black solid circles) from the LV (McCall et al. 2012). Virgo, Fornax, and Hydra SF dwarfs (mostly BCDs) are plotted with black open circles, while TNG Hercules targets having known Arecibo $W_{20}$ line widths are overlaid with green squares. Overall, about half clusters galaxies are located outside the dwarf FP defined by dIs in isolation, showing that the FP relation does not hold for SF dwarfs located in denser environments.

\subsection{Chemical correlations}

Figure 8 plots the well known luminosity - metallicity relation based on the sech magnitude. The dashed line shows the linear fit (Vaduvescu et al. 2007) based on dIs in the LV and Virgo cluster whose oxygen abundances are derived mostly using the precise $T_{\mathrm{e}}$ direct method. Most targets in the four Abell clusters match this linear trend, albeit with larger scatter due to larger uncertainties in Hubble distances and the abundance indirect method (Pilyugin et al. 2010).

Figure 9 draws the metallicity - gas mass relation using mostly $T_{\mathrm{e}}$ oxygen abundance of dwarfs located in the LV and Virgo cluster (Vaduvescu et al. 2007). The TNG targets in Abell 1367 and Hercules having known HI gas masses match this linear relation at the massive end.

Figure 10 shows the metallicity - baryonic mass relation (gas plus stellar mass found from our TNG $K^{\prime}$ data), drawing with a dashed line the linear fit using $28 \mathrm{dIs}$ from LV and Virgo (Vaduvescu et al. 2007). The eight Abell 1367 and Hercules objects appear to confine quite well with this linear trend, probing the fundamental metallicity - mass relation for SF dwarfs.

Figure 11 represents the theoretical correlation assuming a closed box model (Lee et al. 2003) between the oxygen abundance and the gas fraction $\mu$ of SF dwarfs in isolation. From the eight targets with available data, only three galaxies in Hercules agree this model, while the others in Hercules and A1367

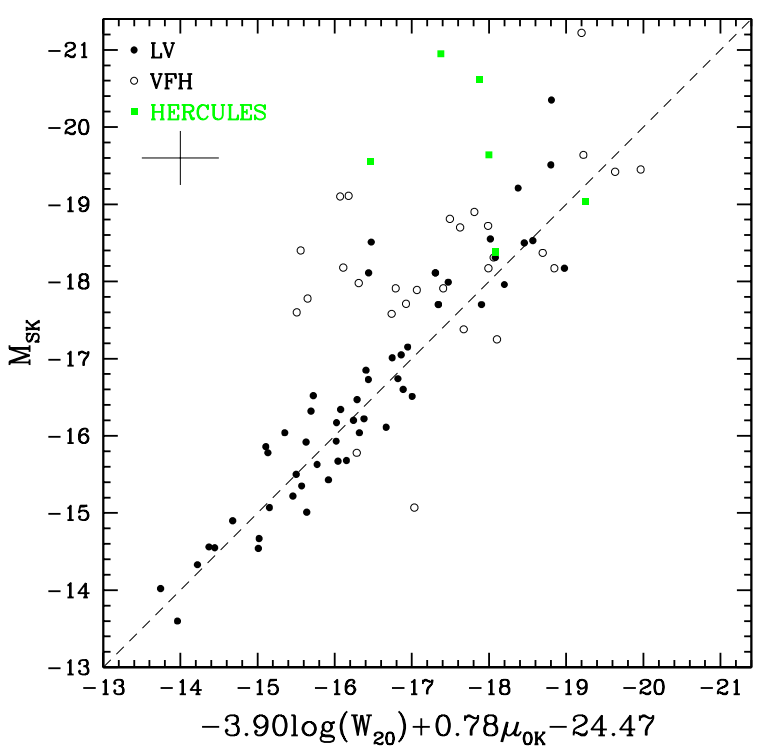

Fig. 7. Fundamental plane (FP) of star forming dwarf galaxies (McCall et al. 2012) defined by 50 dIs from the LV (black solid circles). Formerly studied Virgo, Fornax, and Hydra SF dwarfs (mostly BCDs) are plotted with black open circles, while TNG Hercules targets with available Arecibo data are overlaid with green squares. About half clusters galaxies are located outside the dwarf FP defined by dIs in isolation, suggesting that the FP relation does not hold for SF dwarfs located in denser environments. The cross in the upper left shows typical uncertainties in the two parameters.

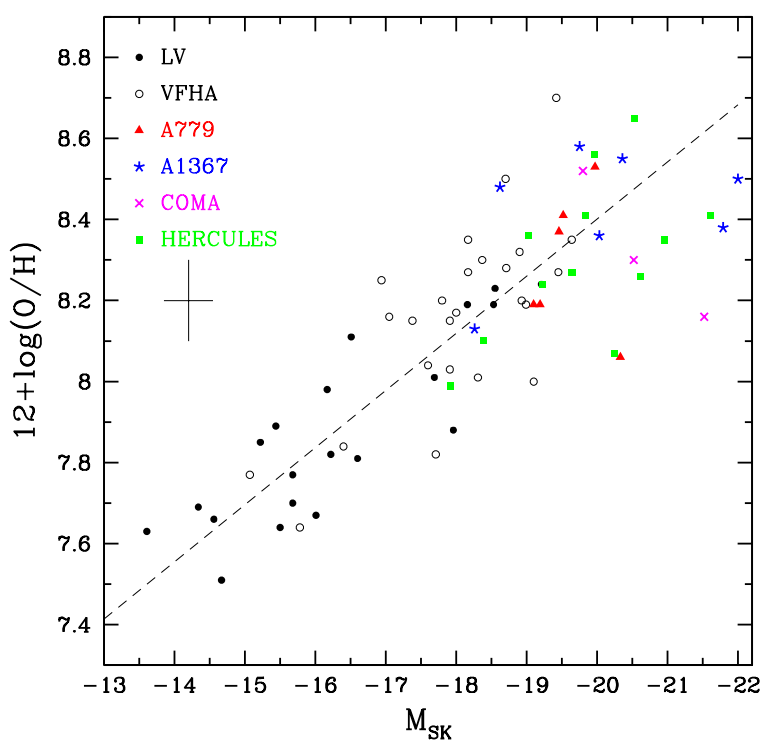

Fig. 8. Luminosity - metallicity relation of SF dwarfs. The dashed line shows the linear fit based on dIs in the LV and Virgo cluster with very precise $T_{\mathrm{e}}$ oxygen abundances (Vaduvescu et al. 2007). Most targets in the four Abell clusters match this linear trend, but have larger scatter due to larger uncertainties in the Hubble distances and the abundance indirect method. The cross in the upper left shows typical uncertainties in the two parameters.

are more faraway. 2151-7583 (IC 1182:[S72] d) is the most evident outlier, known as a tidal dwarf candidate in interaction with IC1182 (Iglesias-Paramo et al. 2003; Petropoulou et al. 2011).

Figure 12 plots the correlation between the logarithm of $\mathrm{H} \alpha$ luminosity (expressed in $\mathrm{erg} \mathrm{s}^{-1}$ and calculated from the $\mathrm{H} \alpha$ flux and the galaxy distances) as a function of the 


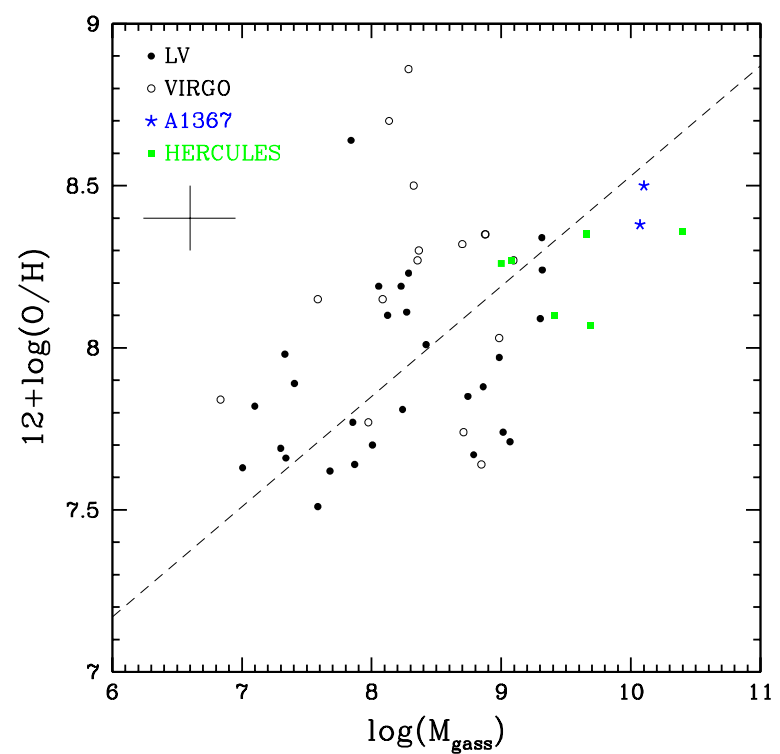

Fig. 9. Metallicity - gas mass relation of SF dwarfs. The dashed line fits dwarfs located in the LV and Virgo cluster, most having precise $T_{\mathrm{e}}$ oxygen abundance (Vaduvescu et al. 2007). The few TNG targets from A1367 and Hercules with available HI gas masses match this linear relation at the massive end. The cross in the upper left shows typical uncertainties in the two parameters.

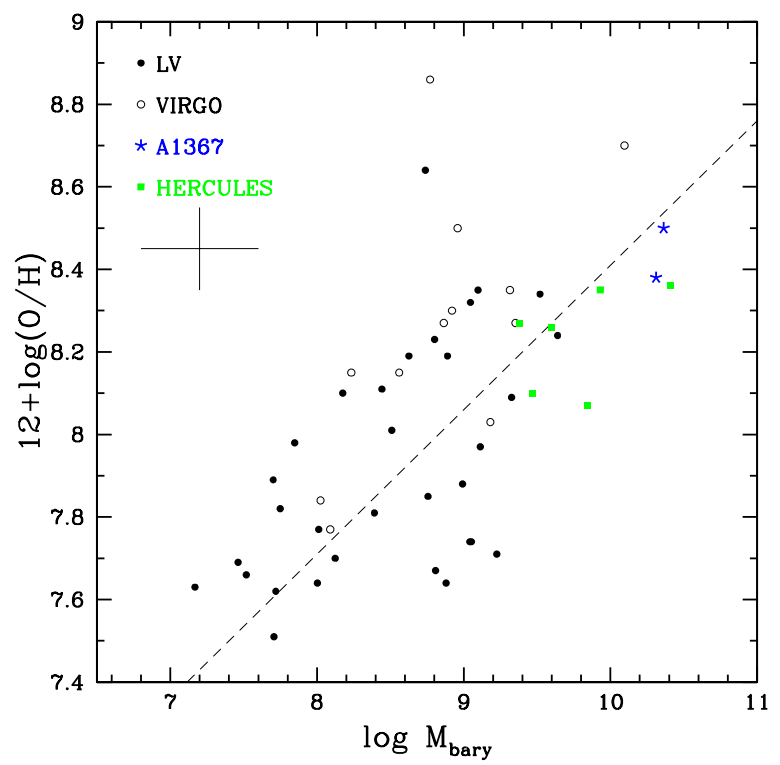

Fig. 10. Metallicity - baryonic (gas and stars) mass relation of SF dwarfs. The dashed line represents linear fit using $28 \mathrm{dIs}$ from LV and Virgo (Vaduvescu et al. 2007). The few A1367 and Hercules objects confine quite well with this linear trend, probing the fundamental metallicity - mass relation for SF dwarfs. The cross in the upper left shows typical uncertainties in the two parameters.

absolute sech magnitude of the objects. The known linear trend published by Cedres et al. (2009) and Iglesias-Paramo et al. (2002) is confirmed, although there is not much improvement going from visible ( $B$ band) to the NIR $\left(K^{\prime}\right)$. The sech magnitude fit $M_{\mathrm{SK}}$ gives better trend than the total magnitude $M_{\mathrm{TK}}$ fit ( $\chi^{2}=0.28$ versus 0.31 ), proving that the sech law in $K$ band is a better gauge of the mass than the total light. We found the following linear fit, plotted with a dashed line

$\log \left(L_{\mathrm{H} \alpha}\right)=-0.30 \times M_{\mathrm{SK}}+34.06$.

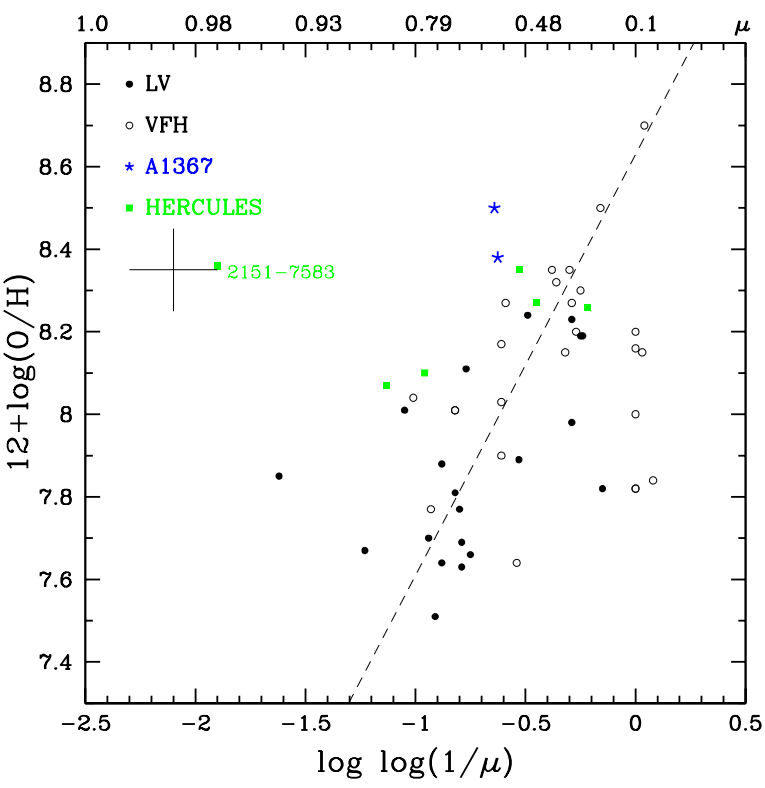

Fig. 11. Metallicity - gas fraction $\mu$ of SF dwarfs, assuming a closed box model (Lee et al. 2003). From the eight Abell TNG targets with available data, only three galaxies in Hercules agree this model, while the others do not. 2151-7583 (IC 1182:[S72] d) is the most evident outlier, known as a tidal dwarf candidate in interaction with IC1182 (Iglesias-Paramo et al. 2003; Petropoulou et al. 2011). The cross in the upper left shows typical uncertainties in the two parameters.

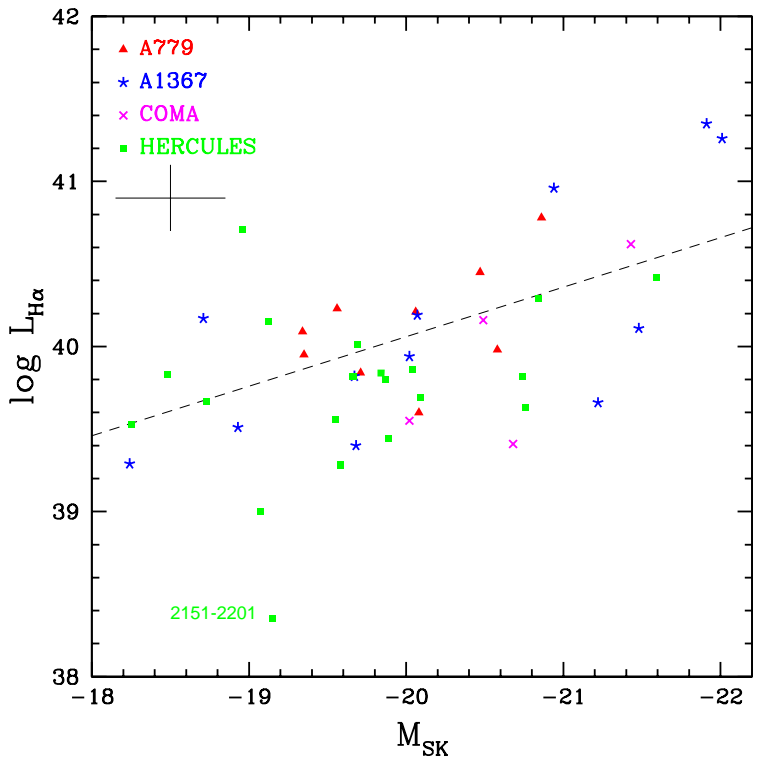

Fig. 12. $\mathrm{H} \alpha$ - luminosity relation of SF dwarfs, expressed as the logarithm of $\mathrm{H} \alpha$ luminosity $\left(\mathrm{erg} \mathrm{s}^{-1}\right)$ as a function of the sech absolute magnitude. The previous linear trend is confirmed (Cedres et al. 2009; Iglesias-Paramo et al. 2002) by the following fit in NIR (Eq. (6), plotted as a dashed line). The cross in the upper left shows typical uncertainties in the two parameters.

\section{Discussion}

\subsection{Sample completeness}

Sample selection is a very important step in observational astronomy and any possible bias needs to be taken into account in the interpretation of the results. There are very few large, deep field surveys aiming to discover dwarf galaxies, as they 
require very large telescopes, dark time and careful image reduction. The popular large field NIR surveys 2MASS, VISTA VHS and UKIDSS LAS are pretty shallow and therefore affected by large photometric errors for dwarfs, as we pointed out in Sect. 4 and our past work (Vaduvescu et al. 2005, 2014). Deep spectroscopic surveys are even more scarce, with the SDSS remaining the main reference and being used in our paper to derive abundances of 17 galaxies in the A779, A1637, and Coma clusters.

Based on the photometric and spectroscopic data from the existing surveys available in 2009 when we planned the TNG observations, probably our galaxy sample is biased towards brighter objects. This fact can be actually observed in Figs. 2 and 3, where most of the TNG targets (plotted with coloured symbols) occupy the brighter part of the plots $\left(M_{\mathrm{SK}} \geq-19 \mathrm{mag}\right)$, in comparison with our previous galaxy samples of SF dwarfs in nearby clusters $\left(-19 \leq M_{\mathrm{SK}} \leq-17 \mathrm{mag}\right.$, plotted with open circles) and with the faintest LV targets $\left(-17 \leq M_{\mathrm{SK}} \leq-13 \mathrm{mag}\right.$, plotted with black solid circles). In consequence, some of the results of this paper are probably biased towards the bright luminosity end in comparison with any theoretical 'complete sample' which should characterise homogeneously the entire SF dwarf population in clusters. This fact was actually expected, due to the increasing difficulty to detect and characterise targets located in more distant clusters in comparison with galaxies observed in the LV (located about 100 times closer than most objects in our sample) or Virgo (residing more than ten times closer).

\subsection{Possible contamination}

We checked the possible contamination of our sample with nondwarf species such as spirals, as suggested nowadays by NED for the following targets in A1367 (1367-7170, 1367-0173, 1367-1370, 1367-4540, 1367-8348, 1367-7325 and 13671014), Coma (1656-9508, 1656-1183 and 1656-0236) and Hercules (2151-3378, 2151-3293, 2151-9062, 2151-8261, 2151-2339, 2151-3041 and 2151-5519). Only three of them (1367-0173, 1656-1183, 2151-5519) are evident outliers labelled in Figs. 2-4.

We also checked the possible contamination of our sample with galaxies actually located outside the clusters. Two Coma targets (1656-5005 and 1656-0236) and seven Hercules targets (2151-5451, 2151-2014, 2151-3378, 2151-2122, 2151-9062, 2151-5482, and 2151-5541) miss velocity data and therefore Hubble distances, and we adopted for them the common cluster distances included in parentheses in the last column of Table 1, leading to physical sizes included in parantheses in Cols. 9 and 10 of Table 2. Only two of these galaxies appear deviant from the past known linear trends (1656-0236 and 2151-2014 labelled in Figs. 2-4).

\subsection{Uncertainties}

The uncertainty in the absolute magnitudes $M_{\mathrm{SK}}$ and $M_{\mathrm{TK}}$ is $\sigma\left(M_{K}\right) \pm 0.35 \mathrm{mag}$. This is composed from the uncertainty in the measured apparent magnitude $(0.2 \mathrm{mag}$ - due to the $2 \mathrm{MASS}$ zero-point $0.1 \mathrm{mag}$ and the photometry errors $0.1 \mathrm{mag}$ ), plus the uncertainty in the galactocentric distance modulus $(0.15 \mathrm{mag}$, according to NED assuming a Hubble flow model). We plot this typical uncertainty in $M_{\mathrm{SK}}$ and $M_{\mathrm{TK}}$ as horizontal error-bars under the legend in Figs. 2-8 and 12.

The uncertainty in the physical size of the galaxies in all four clusters amounts to $14 \%$, being dominated by the uncertainties in the Hubble flow distances (accordingly to NED data). Consequently, the scales (expressed in kpc/arcsec) are affected by the same uncertainty, thus most galaxies with radius $r_{22 \mathrm{~K}}$ between 2-7 kpc (Fig. 2) generate uncertainties between 0.2 and $1.0 \mathrm{kpc}$ with an average of $\sigma\left(r_{22 \mathrm{~K}}\right) \pm 0.6 \mathrm{kpc}$ (plotted as a vertical bar in Fig. 2), and most galaxies with scale lengths between 0.5 and $3.0 \mathrm{kpc}$ generate uncertainties between 0.1 and $0.4 \mathrm{kpc}$ with an average $\sigma\left(r_{0 \mathrm{~K}}\right) \pm 0.25 \mathrm{kpc}$ (plotted as a vertical bar in Fig. 3).

The uncertainty in abundance is $\sigma(12+\log (\mathrm{O} / \mathrm{H}))=0.1 \mathrm{dex}$, according to the bright line method. This is about five times larger than the those of LV galaxies derived from the direct temperature method.

\subsection{Comparison and interpretation}

The size of SF dwarfs from the LV and the nearby clusters has been known to scale with the absolute magnitudes (Vaduvescu et al. 2005, 2006, 2014). This linear trend seems to break above $M_{\mathrm{SK}}=-19$ for targets of more distant clusters, with most galaxies appearing larger at brighter luminosity end (Figs. 2 and 3).

Moreover, the central surface brightness of SF dwarfs from the LV and the nearby clusters has been correlated with the sech absolute magnitudes, but this trend becomes unsustainable for most members of A779, A1367 and especially Hercules (Fig. 4) whose half members with available radio data are clear outliers on our dwarf FP relation well defined in the LV (Fig. 7). These findings actually confirm structural differences obtained in the visible by Barazza et al. (2001) and (Parodi et al. 2002) between field and cluster environments which could be explained by some popular puff up and gas stripping scenarios suggested to take place in denser environmental due to the environmental impact of higher density regions in the clusters.

Despite the evidence of the physical harassment of SF dwarfs, their chemical evolution seems unaffected by denser environments. Albeit the larger metallicity scatter in the bright line method compared with the direct method available for most LV and some Virgo members, the oxygen abundance continues to satisfy the tight linear correlations with the $K$ sech absolute magnitudes (Fig. 8), the gas mass (Fig. 9), the baryonic mass (Fig. 10) and the closed box model (Fig. 11).

\section{Conclusions}

Forty five SF dwarf galaxies located in A779, A1367, A1656 (Coma) and A2151 (Hercules) clusters were imaged with the TNG telescope in the NIR $\left(K^{\prime}\right)$ for four nights in 2010. The clusters and targets were selected based on our past work ( $\mathrm{H} \alpha$ imaging and spectroscopy) and taking into account SDSS DR8 data (available at the time) and the literature.

The SBPs were fitted with a sech component to count the older stellar component at the exterior, plus a Gaussian component to model the inner starburst, proving the BCD classification for most targets. Sech central surface brightness, semimajor axis, sech and total apparent magnitudes were measured, allowing us to extract physical sizes, absolute luminosity and masses, assuming Hubble flow distances taken from NED. Using oxygen abundances, $\mathrm{H} \alpha$ fluxes and some $\mathrm{HI}$ radio data from the literature, we compared the physical and chemical correlations of the observed sample with SF dwarfs previously studied by us in the LV and other nearby clusters (Virgo, Fornax, Hydra and Antlia).

Size $\left(r_{22 S K}\right.$ and $r_{0 S K}$ derived from the sech fit) continues to increase with luminosity ( $\mathrm{sech}$ absolute magnitude $M_{\mathrm{SK}}$ ) but 
the former linear trend is broken around $M_{\mathrm{SK}}=-19$, with more luminous dwarfs in clusters being larger than expected. Central surface brightness $\left(\mu_{0 \mathrm{SK}}\right)$ increases with the absolute magnitude, but the tight linear trend defined in the LV becomes very loose for the members of the four Abell clusters which are fainter at their centre. Colours $\left(m_{r}-m_{\mathrm{TK}}\right)$ vary between 1.5 and $3.5 \mathrm{mag}$, and the outburst excess $\left(m_{\mathrm{SK}}-m_{\mathrm{TK}}\right)$ spread between -0.5 and +0.5 mag, suggesting that sech law fits accurately most targets. The dwarf FP is probed by only half targets with available HI data, while the other half joins other formerly studied cluster data located above the plane.

The oxygen abundances fit linearly with sech absolute magnitude $m_{\mathrm{SK}}$, the logarithm of the gas mass and with the baryonic mass, matching previous results derived in the LV and other clusters, while the closed box model (metallicity versus gas fraction) is probed by most targets, with one exception known as interaction. The starburst (quantified as the logarithm of $\mathrm{H} \alpha$ luminosity) increases with absolute luminosity, matching previous results in visible, but the linear trend remains quite lose.

Acknowledgements. This paper is based on observations made in the Observatorios de Canarias del IAC with the TNG operated on the island of La Palma by the Fundación Galileo Galilei - INAF, Fundación Canaria in the Observatorio del Roque de los Muchachos. OV thanks to the Spanish TAC for the time allocation (programme number CAT_110) during semester 2010A. This research has made use of the NASA/IPAC Extragalactic Database (NED) which is operated by the Jet Propulsion Laboratory, California Institute of Technology, under contract with the National Aeronautics and Space Administration. We used some data and images from Sloan Digital Sky Survey (SDSS, versions DR8 and DR14), which is a project funded by the Alfred P. Sloan Foundation, the U.S. Department of Energy Office of Science, and the Participating Institutions. IRAF is distributed by the National Optical Astronomy Observatory, which is operated by the Association of Universities for Research in Astronomy (AURA) under a cooperative agreement with the National Science Foundation. Thanks are due to the anonymous referee whose recommendations helped us to improve the paper.

\section{References}

Agulli, I., Aguerri, J. A. L., Diaferio, A., et al. 2017, MNRAS, 467, 4410 Barazza, F. D., Binggeli, B., \& Prugniel, P. 2001, A\&A, 373, 12

Cedrés, B., Iglesias-Páramo, J., Vílchez, J. M., et al. 2009, AJ, 138, 873
Cortese, L., Gavazzi, G., Iglesias-Paramo, J., Boselli, A., \& Carrasco, L. 2003, A\&A, 401, 471

Cortese, L., Minchin, R. F., Auld, R. R., et al. 2008, MNRAS, 383, 1519

Coziol, R., Andernach, H., Caretta, C. A., Alamo-Martínez, K. A., \& Tago, E. 2009, AJ, 137, 4795

Hwang, H. S., \& Lee, M. G. 2008, AJ, 676, 218

Iglesias-Páramo, J. 1998, PhD Thesis, Instituto de Astrofísica de Canarias, Spain

Iglesias-Páramo, J., \& Vilchez, J. M. 1997, Rev. Mex. Astron. Astrofis., 6, 263

Iglesias-Páramo, J., \& Vilchez, J. M. 1999, ApJ, 518, 94

Iglesias-Páramo, J., Boselli, A., Cortese, L., Vílchez, J. M., \& Gavazzi, G. 2002, A\&A, 384, 383

Iglesias-Páramo, J., van Driel, W., Duc, P.-A., et al. 2003, A\&A, 406, 453

Karachentsev, I. D., Makarov, D. I., \& Kaisina, E. I. 2013, AJ, 145, 101

Kauffmann, G., White, S. D. M., \& Guiderdoni, B. 1993, MNRAS, 264, 201

Kleinmann, S. G., Lysaght, M. G., Pughe, W. L., et al. 1994, Ap\&SS, 217, 11

Kraiwattanawong, W., James, P. A., Moss, C., \& Carter, D. 2009, ASP Conf. Ser., 404, 39

Lawrence, A., Warren, S. J., Almaini, O., et al. 2007, MNRAS, 379, 1599

Lee, H., McCall, M. L., Kingsburgh, R., Ross, R., \& Stevenson, C. C. 2003, AJ, 125,146

Mahajan, M., Haines, C. P., \& Raychaudhury, S. 2010, MNRAS, 404, 1745

Mahajan, M., Haines, C. P., \& Raychaudhury, S. 2011, MNRAS, 412, 1098

Mateo, M. 1998, ARA\&A, 36, 435

McCall, M. L., Vaduvescu, O., Pozo Nunez, F., et al. 2012, A\&A, 540, 49

Meyer, H. T., Lisker, T., Janz, J., \& Papaderos, P. 2014, A\&A, 562, A49

Parodi, B. R., Barazza, F. D., \& Binggeli, B. 2002, A\&A, 388, 29

Petropoulou, V. 2012, PhD Thesis, Universidad de Granada, Spain

Petropoulou, V., Vílchez, J., Iglesias-Páramo, J., et al. 2011, ApJ, 734, 32

Petropoulou, V., Vílchez, J., \& Iglesias-Páramo, J. 2012, ApJ, 749, 133

Pilyugin, L. S., Vilchez, J. M., \& Thuan, T. X. 2010, ApJ, 720, 1738

Reverte, D. 2008, PhD Thesis, Universidad de Granada, Spain

Reverte, D., Vílchez, J. M., Hernández-Fernández, J. D., \& Iglesias-Páramo, J. 2007, AJ, 133, 705

Thuan, T. X. 1985, ApJ, 299, 881

Vaduvescu, O. 2005, PhD Thesis, York University, ON, Canada

Vaduvescu, O., \& McCall, M. L. 2004, PASP, 116, 640

Vaduvescu, O., \& McCall, M. L. 2008, A\&A, 487, 147

Vaduvescu, O., McCall, M. L., Richer, M. G., \& Fingerhut, R. L. 2005, AJ, 130, 1593

Vaduvescu, O., Richer, M. G., \& McCall, M. L. 2006, AJ, 131, 1318

Vaduvescu, O., McCall, M. L., \& Richer, M. G. 2007, AJ, 134, 604

Vaduvescu, O., Kehrig, C., Vilchez, J. M., \& Unda-Sanzana, E. 2011, A\&A, 533, A65

Vaduvescu, O., Kehrig, C., Bassino, L. P., Smith Castelli, A. V., \& Calderón, J. P. 2014, A\&A, 563, A118

Vilchez, J. M. 1995, AJ, 110, 1090

White, S. D. M., \& Frenk, C. F. 1991, ApJ, 328, 52 


\section{Appendix A: Additional figure}

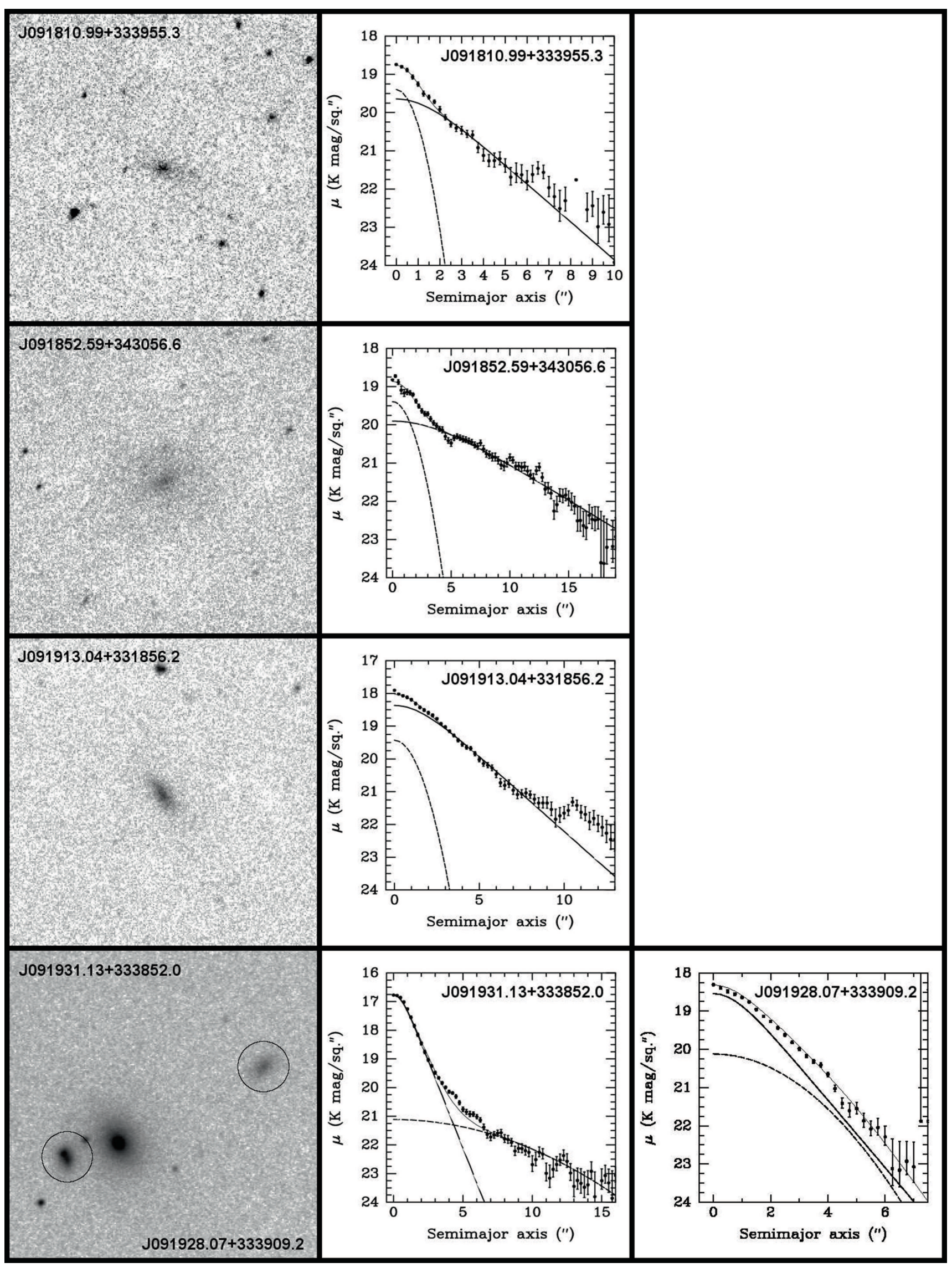

Fig. A.1. TNG reduced $K^{\prime}$ images and surface brightness profiles of the targets. Left: $K^{\prime}$ image field of view $1^{\prime} \times 1^{\prime}$ in normal sky orientation. Right: surface brightness profiles in $K^{\prime}$ fitted by sech law (continuous line) plus Gaussian (dashed line). A779 targets. 


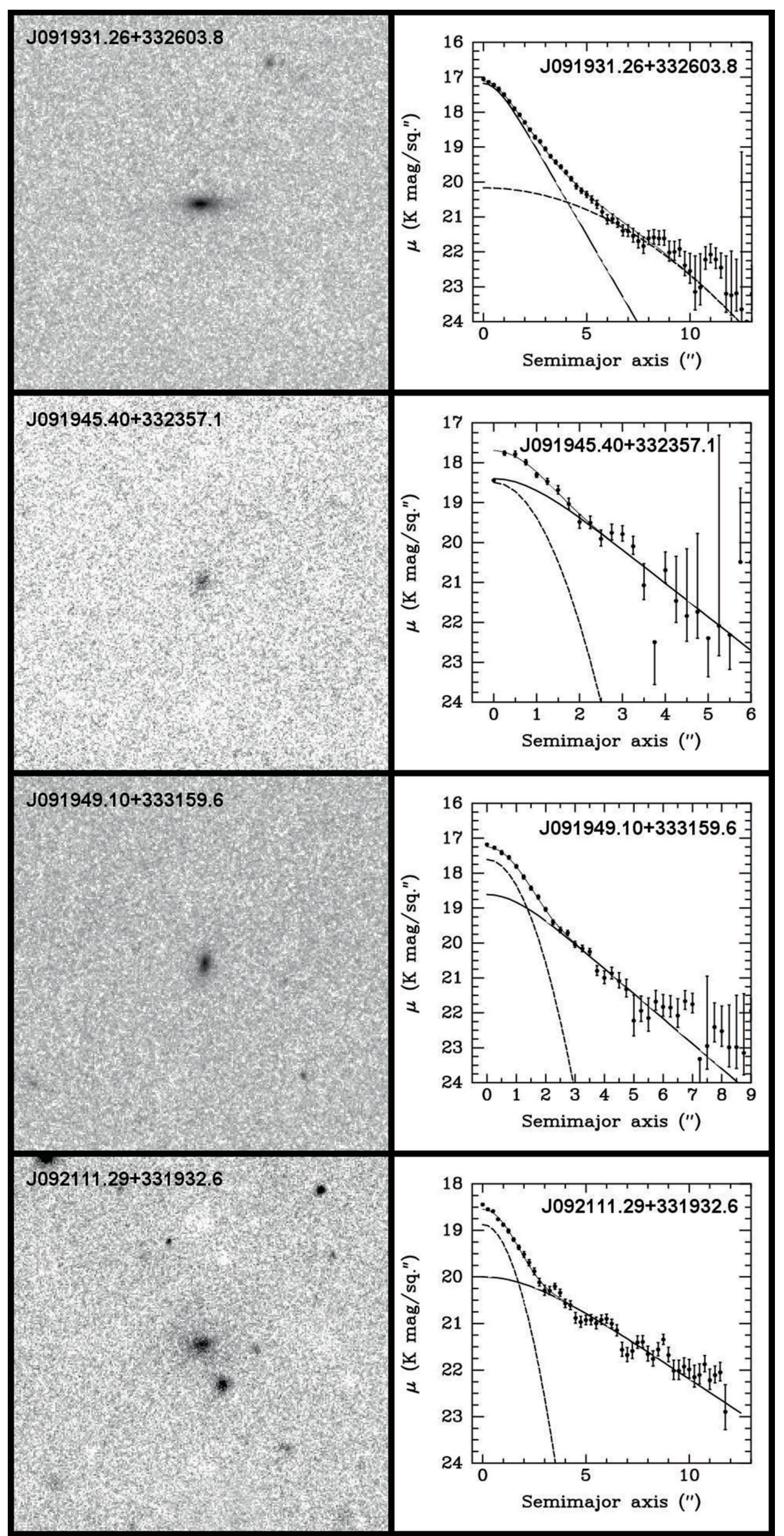

Fig. A.1. continued-A779 targets. 


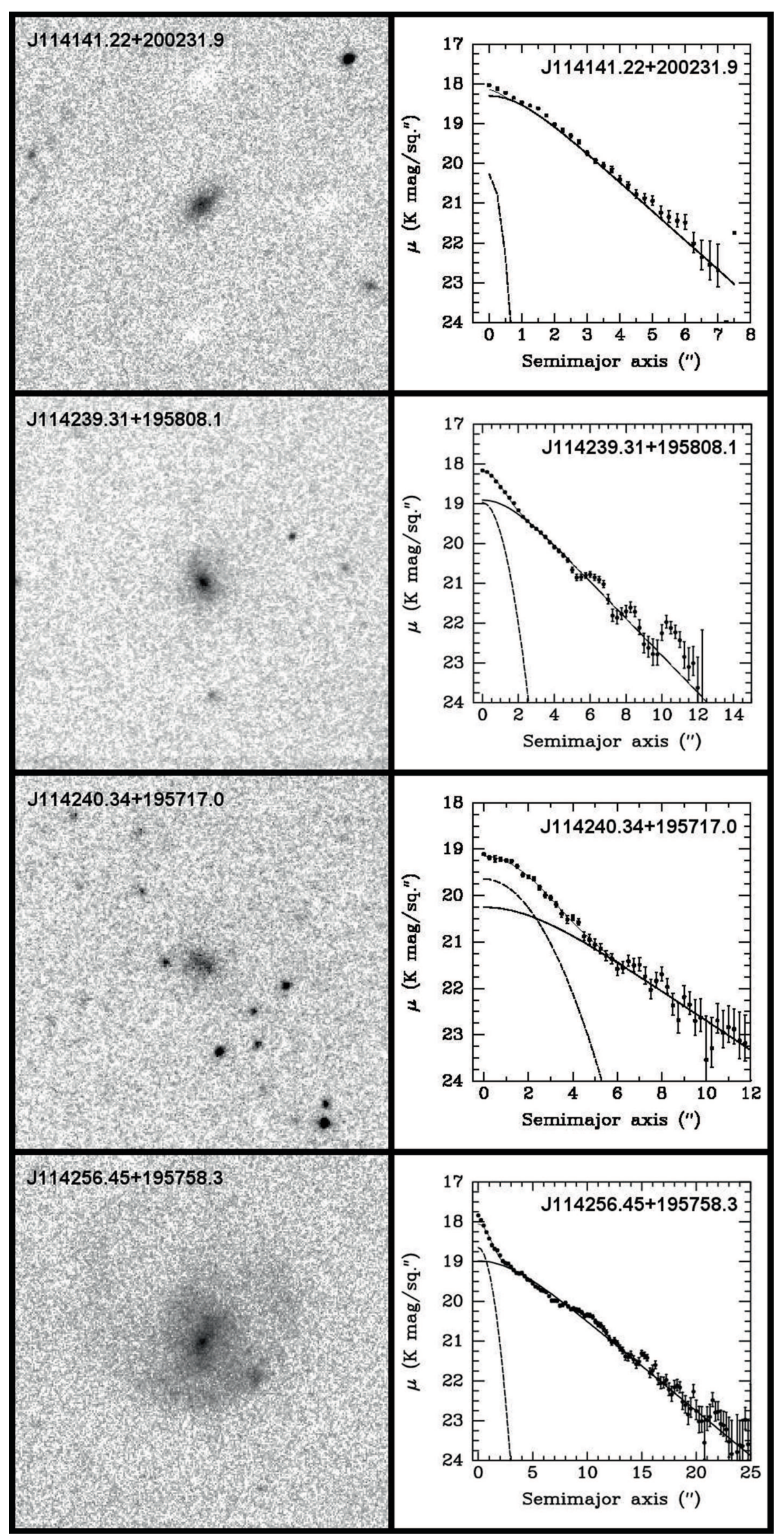

Fig. A.1. continued-A1367 targets. 


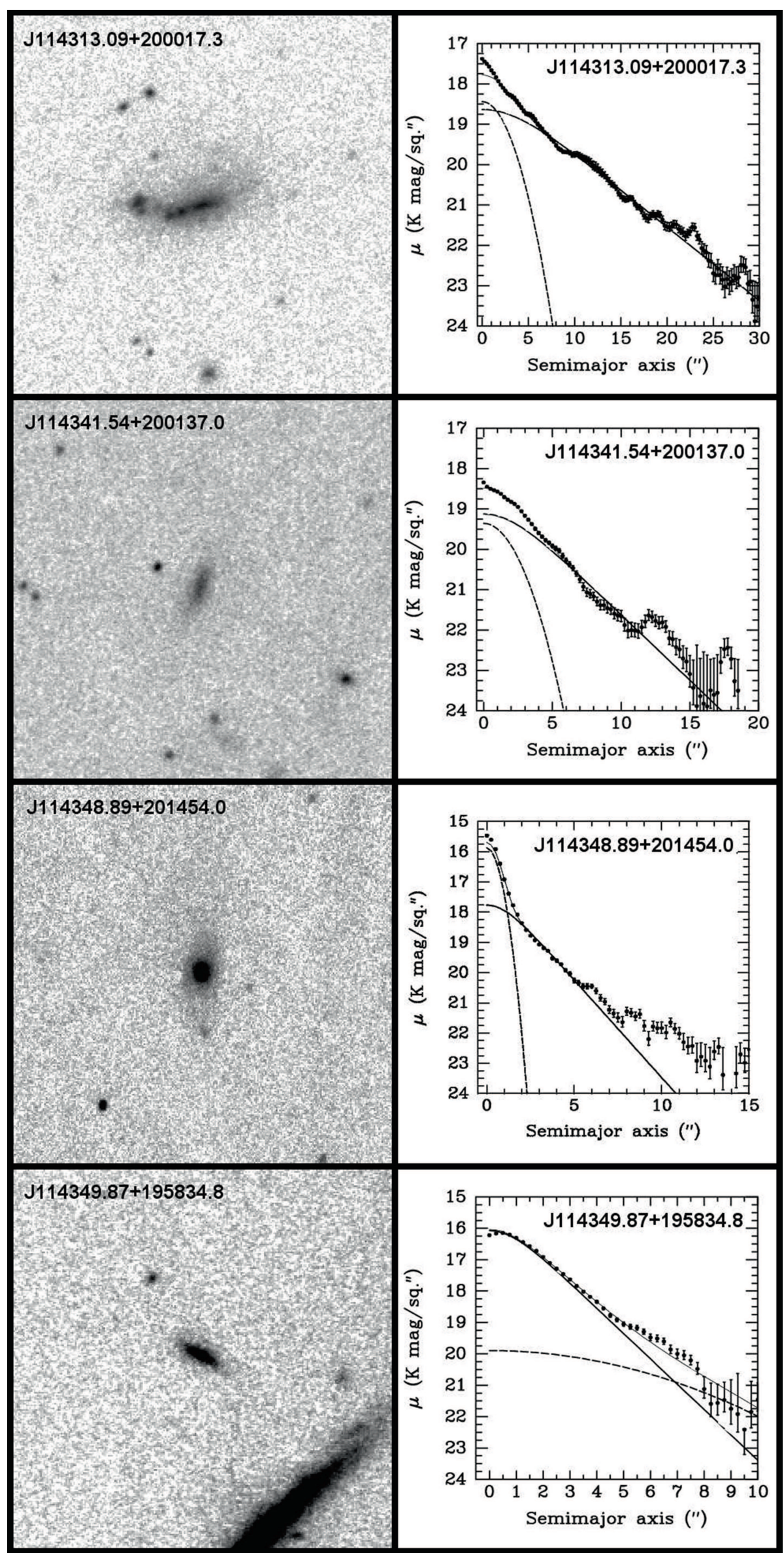

Fig. A.1. continued-A1367 targets. 


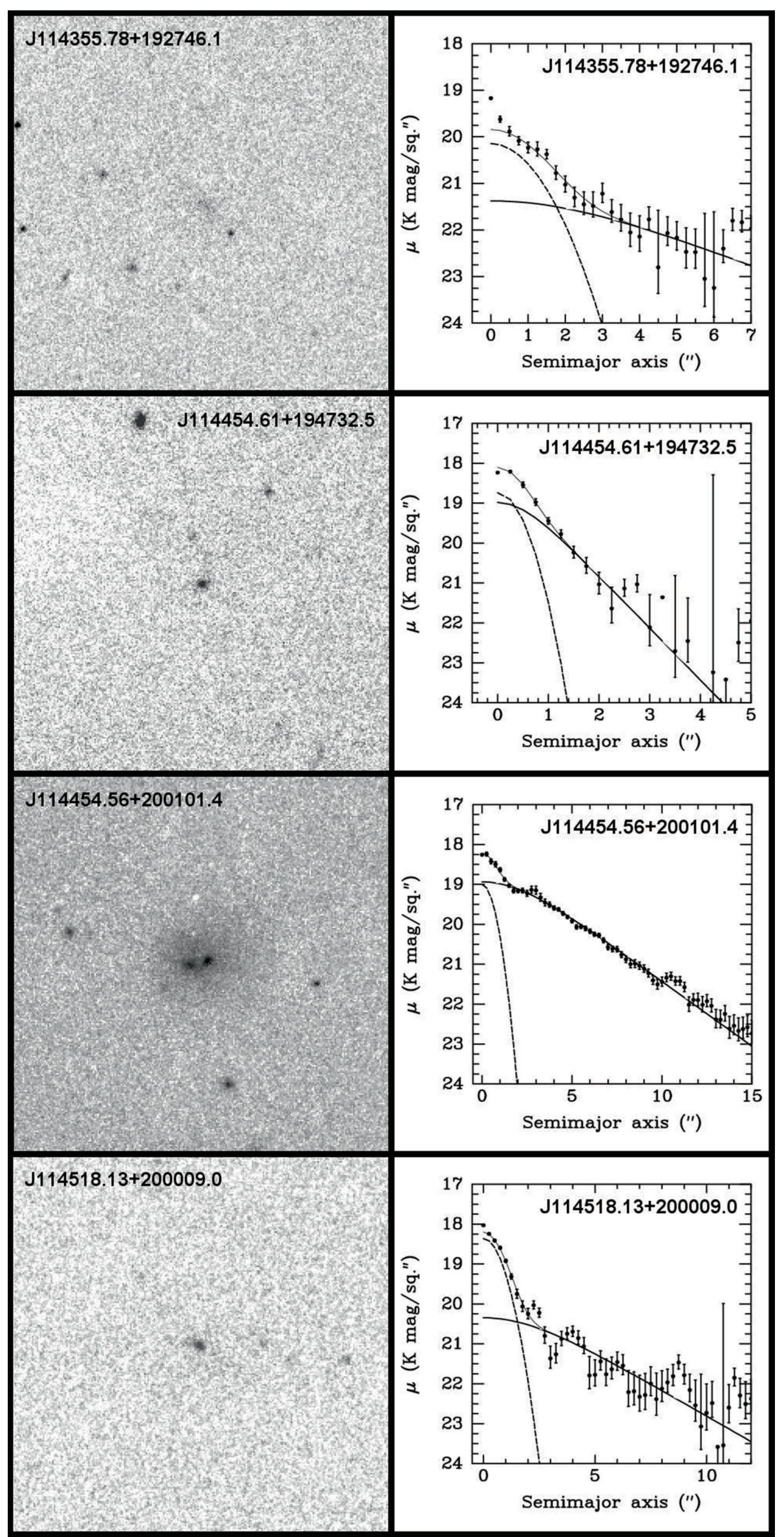

Fig. A.1. continued-A1367 targets. 


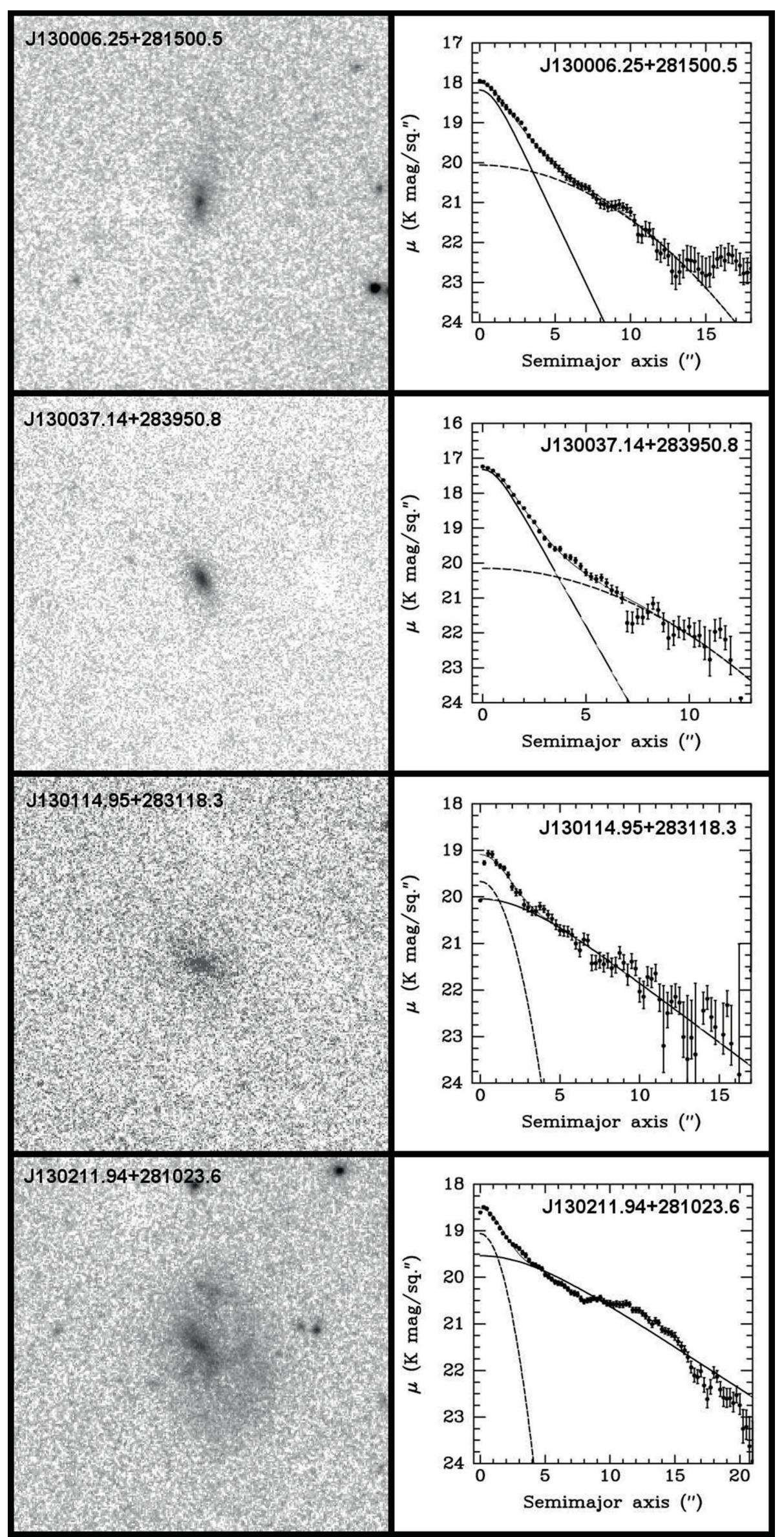

Fig. A.1. continued - A1656 Coma targets. 


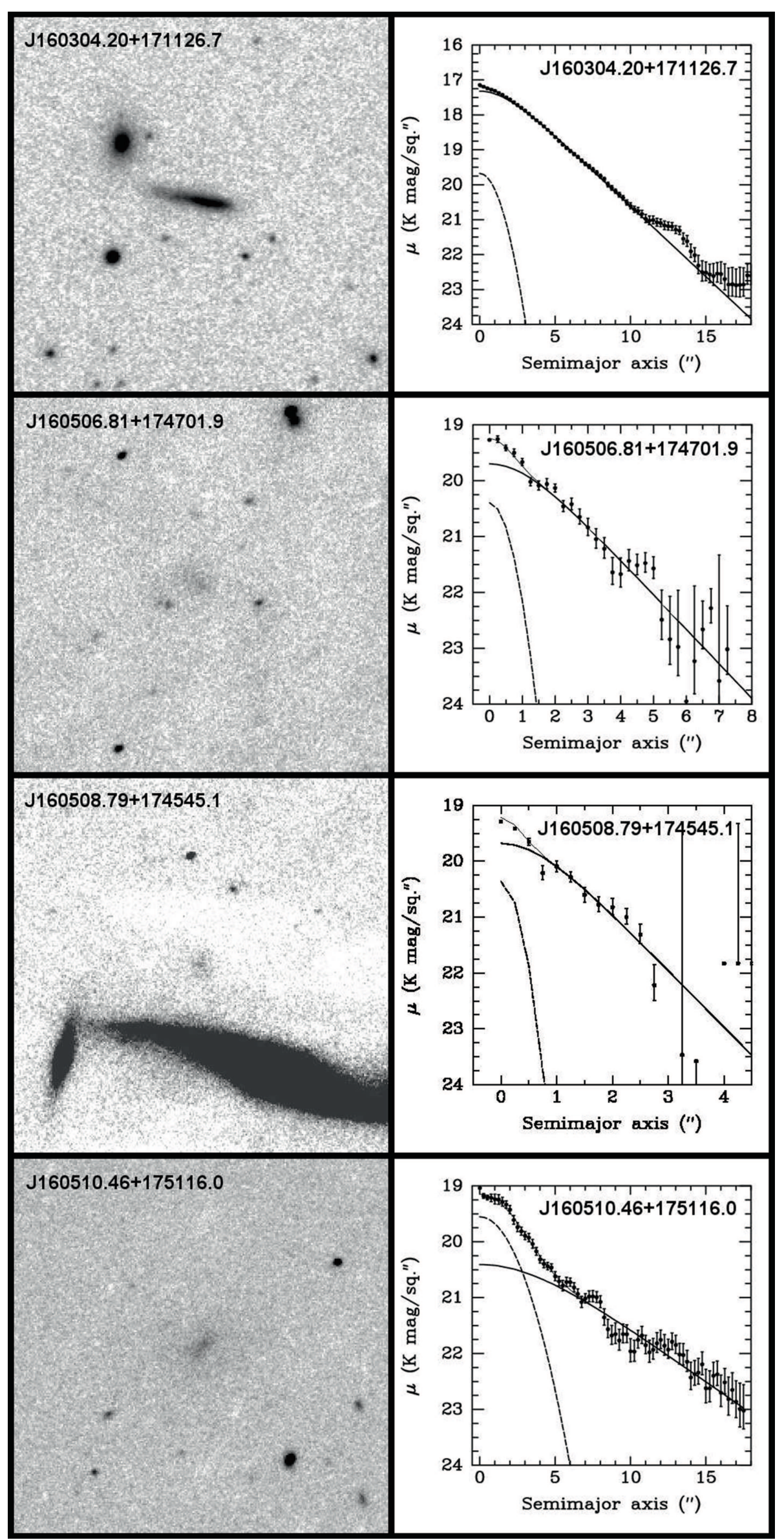

Fig. A.1. continued-A2151 Hercules targets. 


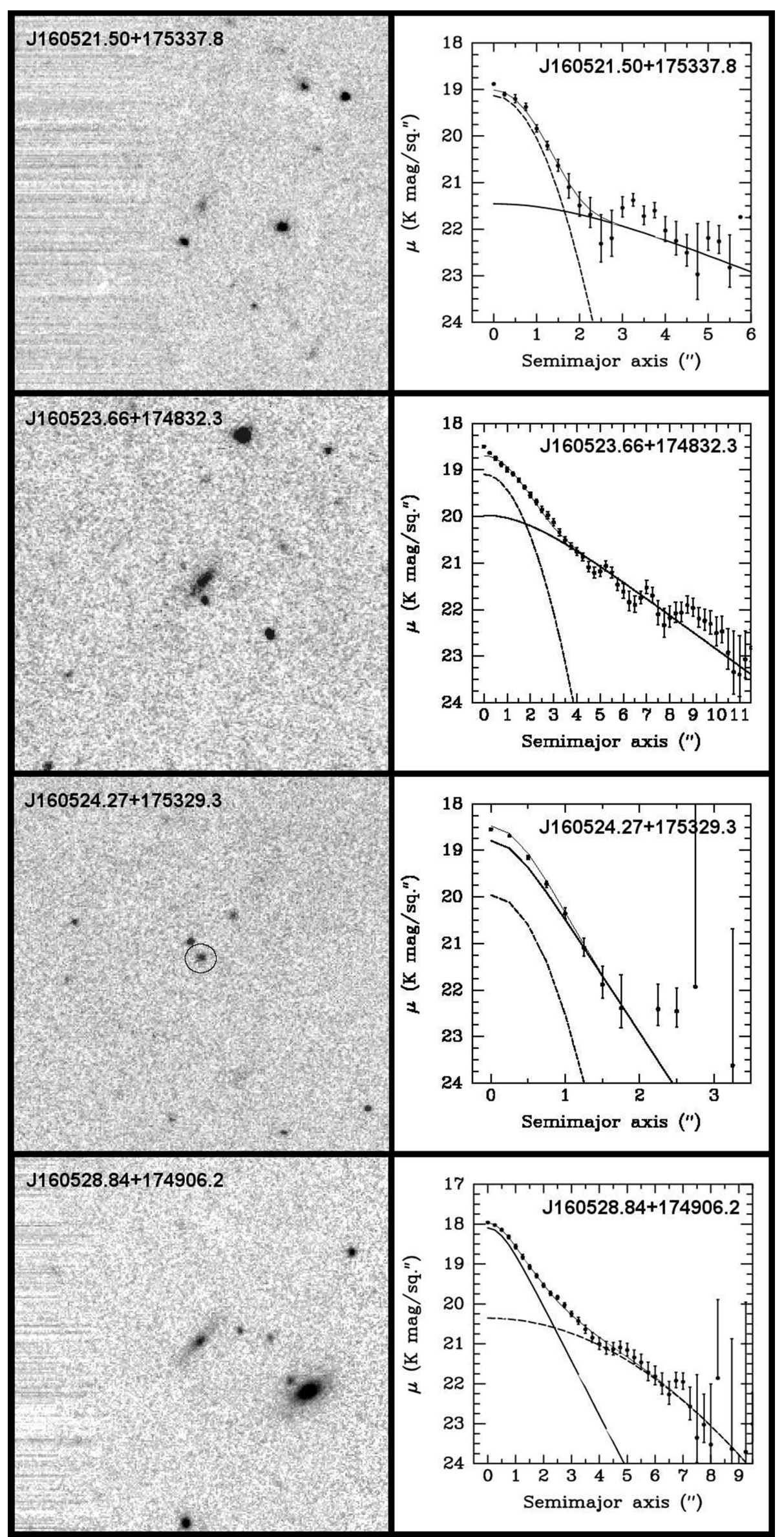

Fig. A.1. continued-A2151 Hercules targets. 


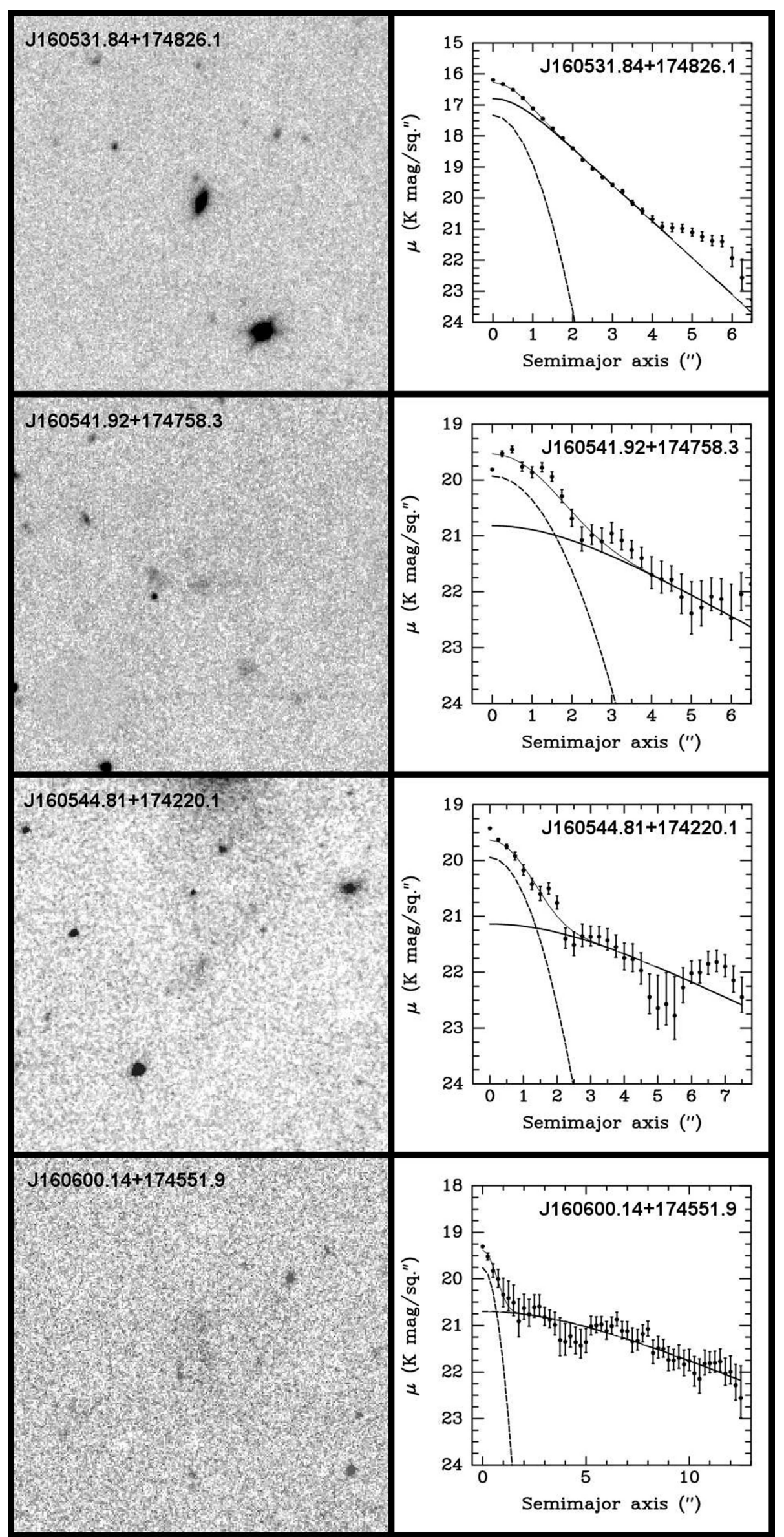

Fig. A.1. continued-A2151 Hercules targets. 


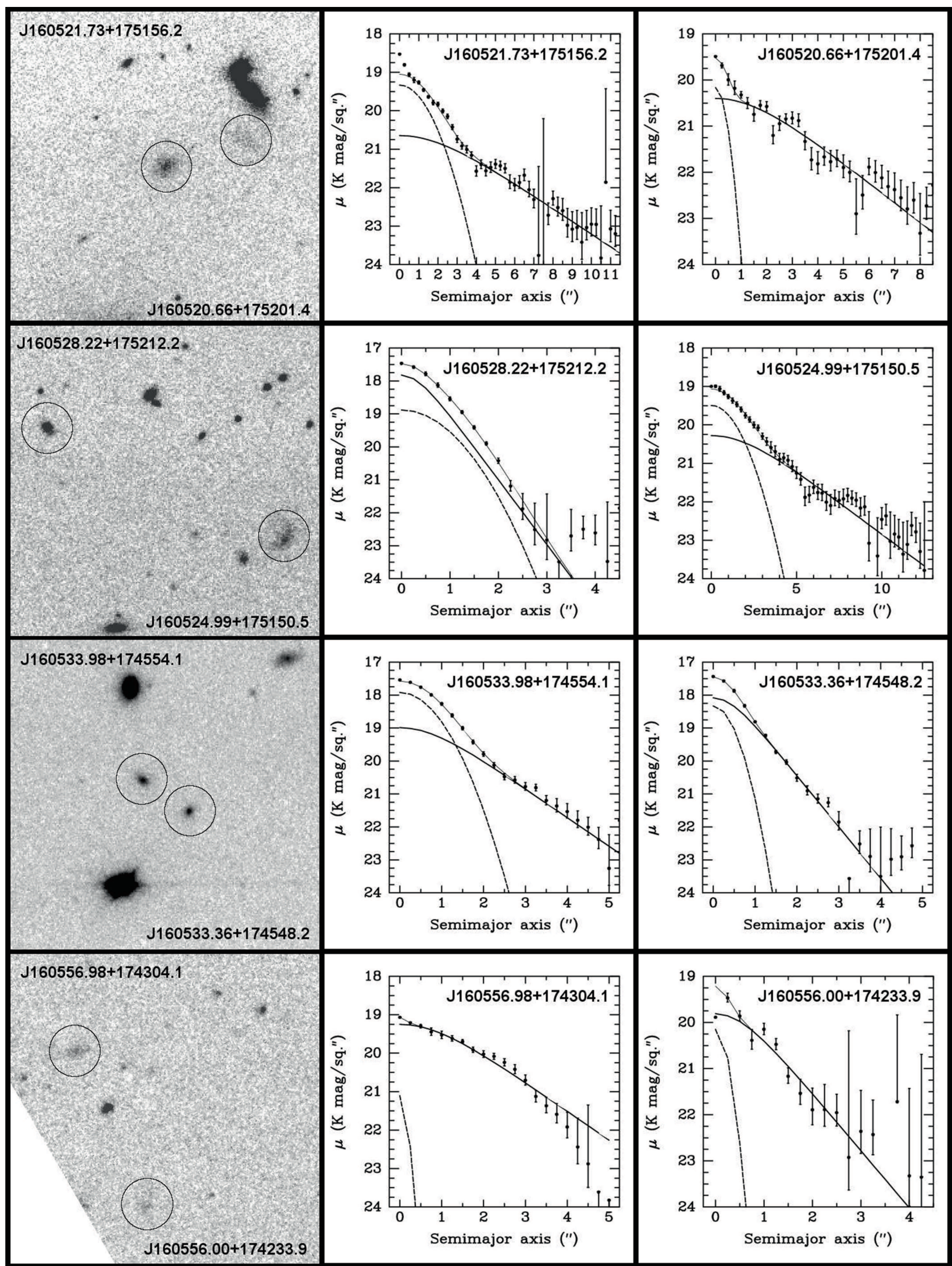

Fig. A.1. continued - A2151 Hercules pairs targets. 